\title{
Noise Tolerance of the BB84 Protocol with Random Privacy Amplification*
}

\author{
Shun Watanabe Ryutaroh Matsumoto \\ Tomohiko Uyematsu \\ Department of Communications and Integrated Systems, \\ Tokyo Institute of Technology, Tokyo 152-8552, Japan
}

June 24, 2005

\begin{abstract}
This paper shows that the BB84 protocol with random privacy amplification is secure with a higher key rate than Mayers' estimate with the same error rate. Consequently, the tolerable error rate of this protocol is increased from $7.5 \%$ to $11 \%$. We also extend this method to the case of estimating error rates separately in each basis, which enables us to securely share a longer key.

Index Terms - Quantum key distribution, BB84, random privacy amplification, security analysis
\end{abstract}

\section{Introduction}

The BB84 protocol is the first quantum key distribution (QKD) protocol, which was proposed by Bennett and Brassard in 1984 1]. Unlike conventional cryptographies that rely on the conjectured difficulty of computing certain functions, the security of QKD is guaranteed by the postulate of quantum mechanics. In the BB84 protocol, the participants (Alice and Bob) agree on a secret key about which any eavesdropper (Eve) can obtain little information. The security proof of this protocol against arbitrary eavesdropping strategies was first proved by Mayers [5], and a simple proof was later shown by Shor and Preskill [2]. Later, many security analyses are studied [6, 7, 8, 14, 15.

In the BB84 protocol, two linear codes $C_{1}$ and $C_{2}$ are employed to share a secret key. $C_{1}$ is used for error correction, and $C_{2}$ is used for privacy amplification. Error correction is performed to share the same key, which is not

\footnotetext{
${ }^{*}$ Part of this paper will be presented in the 2005 International Symposium on Information Theory, Adelaide Convention Centre, Adelaide, Australia, 4-9 September, 2005.
} 
necessarily secret. Privacy amplification is performed to extract a shorter secret key. To share the same secret key, $C_{2}$ must be a subcode of $C_{1}$, and the decoding error probability of $C_{1}$ and $C_{2}^{\perp}$ as a CSS code must be small. For the key distribution protocol to be practical, we require the linear code $C_{1}$ to be efficiently decodeable. However, it is difficult to find a pair of linear codes $C_{1}$ and $C_{2}$ that satisfy these conditions. Because we do not have to decode $C_{2}^{\perp}$, it is sufficient that $C_{2}^{\perp}$ is a randomly chosen code whose decoding error probability is small with the maximum likelihood decoding. Mayers showed that if one determines $C_{1}$ and chooses $C_{2}$ with rate $\mathrm{H}(2 p)$ at random from subcodes of $C_{1}$, the minimum Hamming weight of $C_{2}^{\perp} \backslash C_{1}^{\perp}$ is greater than $p n$ with high probability, where $p$ is an estimated error rate and $\mathrm{H}(\cdot)$ is the binary entropy function [5, Lemma 4]. Consequently, the decoding error probability of $C_{2}^{\perp}$ as a part of a CSS code is small. With this method, we can share a key with the key rate $1-\mathrm{H}(p)-\mathrm{H}(2 p)$, and the protocol can tolerate error rates up to $7.5 \%$. In this paper, we call the random privacy amplification as the method such that one chooses $C_{2}$ at random from subcodes of a fixed code $C_{1}$ and performs the privacy amplification by $C_{2}$.

However, by evaluating directly the decoding error probability of $C_{2}^{\perp}$ instead of the minimum Hamming weight, we can decrease the rate of $C_{2}$ while maintaining the security of the protocol. This paper shows that when one chooses $C_{2}$ with rate $\mathrm{H}(p)$ at random from subcodes of $C_{1}$, the decoding error probability of $C_{2}^{\perp}$ as a part of a CSS code is exponentially small with high probability. Consequently, when we choose a code $C_{2}$ at random in the BB84 protocol, according to our evaluation of decoding error probability, we can share a key with the key rate $1-2 \mathrm{H}(p)$ and the protocol can tolerate error rates up to $11 \%$.

To share a key more efficiently, it is known that we should estimate error rates, $p_{0}$ and $p_{1}$, separately in two basis $\{|0\rangle,|1\rangle\}$ and $\left\{\frac{|0\rangle+|1\rangle}{\sqrt{2}}, \frac{|0\rangle-|1\rangle}{\sqrt{2}}\right\}$ [6] [7] 8. 14. This paper also shows that if one chooses $C_{2}$ of a rate $\frac{\mathrm{H}\left(p_{0}\right)+\mathrm{H}\left(p_{1}\right)}{2}$ at random from subcodes of $C_{1}$, the decoding error probability of $C_{2}^{\perp}$ as a part of a CSS code is exponentially small with high probability, which is proved by an analogue method in [6] Appendix B].

It is also known that QKD protocols with two-way classical communications can tolerate higher error rate than QKD protocols with one-way classical communications. The tolerable error rates are $18.9 \%$ in [7, $20 \%$ in [1], and $26 \%$ in 10. Our result on random privacy amplification is also applicable for those protocols, because they perform error correction and privacy amplification after reducing the error rate with two-way classical communications.

We stress that our result is different from previously known results. In 5 , Lemma 4], it is proved that if we fix $C_{1}$ of rate $1-\mathrm{H}(p)$ and choose its subcode $C_{2}$ of rate $\mathrm{H}(2 p)$ at random, the $\mathrm{BB} 84$ protocol is secure, which means that the $\mathrm{BB} 84$ protocol with random privacy amplification can tolerate the error rate of $7.5 \%$. In [2], the authors state that there exists a pair of $C_{1}$ and $C_{2}$ by which the BB84 protocol can tolerate the error rate of $11 \%$. However, one cannot guarantee that $C_{1}$ is efficiently decodeable. They also cite [5, Lemma 4] in order to show that we can securely choose a random subcode $C_{2}$ of an 
efficiently decodeable code $C_{1}$. However, it is not clarified in [2] whether or not the BB84 protocol with random privacy amplification can tolerate the $11 \%$ error rate. Other previous papers [6, 7, 8, 14, 15] are based on the result in 2. Thus nobody has proved that the BB84 protocol with random privacy amplification can tolerate the $11 \%$ error rate. We also stress that the random hashing method cannot be directly applied to the security proof of the BB84 protocol with random privacy amplification as used in [16], because a fixed $C_{1}$ and the condition $C_{2} \subset C_{1}$ decrease the randomness of hashing. Application of the random hashing to a security proof of the random privacy amplification requires a careful argument similar to Section 3 of this paper.

This paper is organized as follows. In Section [2 we introduce the BB84 protocol, and present the required conditions on $C_{1}$ and $C_{2}$. We also relate those conditions to the security of the BB84 protocol quantitatively. In Section [3 the main theorem is proved. Concluding remarks are given in Section 4

\section{The BB84 protocol}

We consider the following BB84 protocol modified from [2]. As shown in 66. 7] 8, 14, we estimate error rates separately in two basis $\{|0\rangle,|1\rangle\}$ and $\left\{\frac{|0\rangle+|1\rangle}{\sqrt{2}}, \frac{|0\rangle-|1\rangle}{\sqrt{2}}\right\}$. As is also mentioned in [2] 6, 7], Alice and Bob agree on a random permutation $\pi$ after transmission of the qubits and use the linear codes scrambled by $\pi$, where $\pi$ scrambles the $n$-bit vector within first $\frac{n}{2}$ bits and latter $\frac{n}{2}$ bits respectively, i.e., $\pi:\left(x_{1}, \cdots, x_{\frac{n}{2}}, y_{1}, \cdots, y_{\frac{n}{2}}\right) \mapsto\left(x_{\pi_{1}(1)}, \cdots, x_{\pi_{1}\left(\frac{n}{2}\right)}, y_{\pi_{2}(1)}\right.$, $\left.\cdots, y_{\pi_{2}\left(\frac{n}{2}\right)}\right)$, and $\pi_{1}, \pi_{2} \in S_{\frac{n}{2}}$ are permutations on $\left\{1, \cdots, \frac{n}{2}\right\}$. By this procedure, we can securely share a key against general eavesdropping attacks with a linear code whose decoding error probability as a part of a CSS code is small over a BSC (Binary Symmetric Channel) [7, Lemmas 2, 3].

\subsection{The BB84 protocol}

(1) Alice randomly select $(4+\theta) n$-bit strings $\boldsymbol{k}$ and $\boldsymbol{a}$, and chooses a random permutation $\pi$.

(2) Alice repeats the following procedures for $1 \leq i \leq(4+\theta) n$. If $a_{i}=0$, she creates either state $|0\rangle$ for $k_{i}=0$ or $|1\rangle$ for $k_{i}=1$. If $a_{i}=1$, she creates either state $|+\rangle$ for $k_{i}=0$ or $|-\rangle$ for $k_{i}=1$. We represent prepared states as $\left|\varphi_{i}\right\rangle$, where $\varphi_{i} \in\{0,1,+,-\},|+\rangle=\frac{|0\rangle+|1\rangle}{2},|-\rangle=\frac{|0\rangle-|1\rangle}{2}$.

(3) Alice sends the resulting $(4+\theta) n$ qubits $\left|\varphi_{1}\right\rangle \otimes \cdots \otimes\left|\varphi_{(4+\theta) n}\right\rangle$ to Bob.

(4) Bob receives the $(4+\theta) n$ qubits $\left|\tilde{\varphi}_{1}\right\rangle \otimes \cdots \otimes\left|\tilde{\varphi}_{(4+\theta) n}\right\rangle$.

(5) Bob randomly select $(4+\theta) n$-bit string $\boldsymbol{b}$.

(6) Bob repeats the following procedures for $1 \leq i \leq(4+\theta) n$. If $b_{i}=0$, he measures $\left|\tilde{\varphi}_{i}\right\rangle$ with $\sigma_{z}$. If $b_{i}=1$, he measures $\left|\tilde{\varphi}_{i}\right\rangle$ with $\sigma_{x}$. Then, measure- 
ment result, +1 and -1 , corresponds to $\tilde{k}_{i}=0$ and $\tilde{k}_{i}=1$, respectively. After these procedures Bob will obtain $\tilde{\boldsymbol{k}}=\left(\tilde{k}_{1}, \cdots, \tilde{k}_{(4+\theta) n}\right)$.

(7) Alice announces $\boldsymbol{a}$ and $\pi$.

(8) If $a_{i} \neq b_{i}$, Alice and Bob discard $i$-th bit of $\boldsymbol{k}$ and $\tilde{\boldsymbol{k}}$. With high probability, at least $2 n$ bits remain, and there are at least $n$ bits where $a_{i}=b_{i}=0$, and there are at least $n$ bits where $a_{i}=b_{i}=1$ (if not, abort the protocol).

(9) Alice chooses $n$ bits where $a_{i}=b_{i}=0$, and divides them into two $\frac{n}{2}$-bit strings, $\boldsymbol{c}_{0}$ and $\boldsymbol{d}_{0}$. She chooses $n$ bits where $a_{i}=b_{i}=1$, and divides them into two $\frac{n}{2}$-bit strings, $\boldsymbol{c}_{1}$ and $\boldsymbol{d}_{1}$. Alice announces which bits are $\boldsymbol{c}_{0}, \boldsymbol{d}_{0}$, $\boldsymbol{c}_{1}, \boldsymbol{d}_{1}$. Then, Bob will obtain $\tilde{\boldsymbol{c}}_{0}=\boldsymbol{c}_{0}+\boldsymbol{e}_{0}, \tilde{\boldsymbol{c}}_{1}=\boldsymbol{c}_{1}+\boldsymbol{e}_{1} \tilde{\boldsymbol{d}}_{0}=\boldsymbol{d}_{0}+\boldsymbol{f}_{0}$, $\tilde{\boldsymbol{d}}_{1}=\boldsymbol{d}_{1}+\boldsymbol{f}_{1}$, where $\boldsymbol{e}_{0}, \boldsymbol{e}_{1}, \boldsymbol{f}_{0}, \boldsymbol{f}_{1}$ are errors caused by eavesdropping and channel noise.

(10) Alice and Bob compare $\boldsymbol{d}_{0}$ with $\tilde{\boldsymbol{d}}_{0}$ and $\boldsymbol{d}_{1}$ with $\tilde{\boldsymbol{d}}_{1}$, then they obtain $\boldsymbol{f}_{0}$ and $\boldsymbol{f}_{1}$. From $\boldsymbol{f}_{0}, \boldsymbol{f}_{1}$, Alice and Bob choose a pair of linear codes $C_{1}$ and $C_{2}$ that satisfy the conditions (aㅣ) $-(\mathbb{a})$ in Section 2.2 If there exists no such a pair of linear codes, then they abort the protocol.

(11) Alice chooses a random codeword $\boldsymbol{v}$ from $\pi\left(C_{1}\right)$ whose length is $n$, where $\pi\left(C_{1}\right)$ is a code that all codewords in $C_{1}$ are permuted by $\pi$. She sends $\boldsymbol{x}=\boldsymbol{v}+\boldsymbol{c}$ with a public classical channel, where $\boldsymbol{c}$ is a concatenation of $\boldsymbol{c}_{0}$ and $\boldsymbol{c}_{1}$.

(12) Bob receives $\boldsymbol{x}=\boldsymbol{v}+\boldsymbol{c}$ and subtracts $\tilde{\boldsymbol{c}}$ from it. Then, he corrects $\boldsymbol{v}+\boldsymbol{e}$ to a codeword $\hat{\boldsymbol{v}}$ in $\pi\left(C_{1}\right)$, where $\boldsymbol{e}$ is a concatenation of $\boldsymbol{e}_{0}$ and $\boldsymbol{e}_{1}$, and $\tilde{\boldsymbol{c}}$ is a concatenation of $\tilde{\boldsymbol{c}}_{0}$ and $\tilde{\boldsymbol{c}}_{1}$.

(13) Alice uses the coset of $\boldsymbol{v}+\pi\left(C_{2}\right)$ as a key, and Bob uses the coset of $\hat{\boldsymbol{v}}+\pi\left(C_{2}\right)$ as a key.

\subsection{Security of the protocol}

The security of the BB84 protocol can be proved by showing the security of the CSS code protocol (QKD using a CSS code) 6]. Maintaining the security, the BB84 protocol is related with the CSS code protocol. This kind of technique was first used in 2], in which the BB84 protocol is related to the EPP (Entanglement Purification Protocol) protocol. If $C_{1}$ and $C_{2}$ satisfy the following three conditions, then a shared key is secure against general eavesdropping attacks.

(a) $C_{2} \subset C_{1}$

(b) If the crossover probability of first $\frac{n}{2}$ bits of the BSC are smaller than or equal to $p_{0}$ and the crossover probability of latter $\frac{n}{2}$ bits are smaller than or equal to $p_{1}$, then the decoding error probability of $C_{1}$ as a part of a CSS code over the BSC, whose formal definition is given in Definition 2 is smaller than or equal to $\epsilon$. 
(c) If the crossover probability of first $\frac{n}{2}$ bits of the BSC are smaller than or equal to $p_{1}$ and the crossover probability of latter $\frac{n}{2}$ bits are smaller than or equal to $p_{0}$, then the decoding error probability of $C_{2}^{\perp}$ as a part of a CSS code over the BSC is smaller than or equal to $\epsilon$.

We set $p_{0}$ and $p_{1}$ to $p_{0}=Q_{\boldsymbol{f}_{0}}(1)+\delta$ and $p_{1}=Q_{\boldsymbol{f}_{1}}(1)+\delta$ in step (10), where $Q_{\boldsymbol{f}_{0}}, Q_{\boldsymbol{f}_{1}}$ are the types of $\boldsymbol{f}_{0}, \boldsymbol{f}_{1}$ (refer to 9 ] for the definition of the type), and $\delta$ and $\epsilon$ are sufficiently small positive numbers. Throughout this paper, we assume $p_{0}<\frac{1}{2}$ and $p_{1}<\frac{1}{2}$.

We stress that the decoding error probability of $C_{1}$ and $C_{2}^{\perp}$ have to be small over any BSC with crossover probability below $p_{0}$ and crossover probability below $p_{1}$, instead of a single BSC with crossover probabilities $p_{0}$ and $p_{1}$. The necessity of such a requirement on decoding error probability is already observed in [6], 7] Proof of Lemma 3].

The security of the BB84 protocol is usually evaluated by the mutual information between a shared key and Eve's accessible information. In order to implement the BB84 protocol, the designer of the system has to find a pair of linear codes by which the mutual information between a shared key and Eve's accessible information is smaller than an acceptable level. To find such a pair of linear codes, we need a criterion according to which we can distinguish whether a particular pair of linear codes makes the mutual information smaller than an acceptable level.

In the security proof of [7, it is proved that the security of the BB84 protocol against general eavesdropping attack is reduced to the security against uncorrelated Pauli attacks (Eve applies a random Pauli operator independently on each qubit sent through the channel). However, only a asymptotic upper bound on the mutual information is proved, and the authors do not present a sufficient condition for low mutual information on a pair of linear codes of a finite code length.

In the security proof of [6], Hamada presents a condition on a pair of linear codes [6. Corollary 2], and proves that the mutual information is upper bounded quantitatively by a pair of linear codes satisfying that condition. However, that condition does not aid choosing a suitable linear code, because we cannot easily decide whether a particular code satisfies it.

By upper bounding the mutual information by a function of the decoding error probability $\epsilon$, we can find a pair of linear codes that makes the mutual information smaller than an acceptable level according to the conditions (国) -(远). Because evaluating an upper bound on the decoding error probability of a code is not difficult, the conditions ( $(\mathbf{a})-(\mathbb{C})$ on a pair of linear codes are practically useful. The following theorem gives an upper bound on the mutual information as a function of the decoding error probability $\epsilon$.

Theorem 1 If we use linear codes $C_{1}$ and $C_{2}$ that satisfy the conditions (a)(c) in the BB84 protocol, then the mutual information between a shared key and Eve's accessible information (including messages exchanged over the classical 
channel) is upper bounded by

$$
\begin{aligned}
& \mathrm{I}(\boldsymbol{U} ; \boldsymbol{E}, \boldsymbol{S}) \\
\leq & \mathrm{H}\left(2\left(\frac{n}{2}+1\right)^{2} \epsilon+2 \exp \left\{-\Theta\left(\delta^{2} n\right)\right\}\right)+4 n\left(\frac{n}{2}+1\right)^{2} \epsilon+4 n \exp \left\{-\Theta\left(\delta^{2} n\right)\right\},
\end{aligned}
$$

where the base of $\exp (\cdot)$ is $2, \Theta\left(\delta^{2} n\right)$ is given by

$$
\Theta\left(\delta^{2} n\right)=\frac{\delta^{2}}{4 \ln 2} n-2 \log (n+1)-2,
$$

$\boldsymbol{S}$ denotes the random variable of the information transmitted through the public classical channel, $\boldsymbol{U}$ denotes the random variable of a shared key, i.e., the coset of $\boldsymbol{v}+\pi\left(C_{2}\right)$, and $\boldsymbol{E}$ denotes the random variable of Eve's eavesdropping result from transmitted qubits.

This theorem is proved in Appendix A Note that the upper bound of the mutual information is valid for finite $n$.

\section{Random privacy amplification}

To implement the BB84 protocol, we need a linear code $C_{1}$ to be efficiently decodeable, which is used for error correction in step (12). Under the conditions (国) -(IC), it is difficult to find a pair of linear codes $C_{1}$ and $C_{2}$ of which $C_{1}$ is efficiently decodeable. On the other hand, since we do not decode $C_{2}^{\perp}$ in the BB84 protocol, we can evaluate the condition (c) with an arbitrary decoding method. Therefore, first we choose a code $C_{1}$ that satisfies the condition (b) and is efficiently decodeable. Then we will find a code $C_{2}$ that satisfies the conditions (a) and (ㄷ). Given a code $C_{1}$, choosing a code $C_{2}$ with the condition (aid) is same as choosing a code $C_{2}^{\perp}$ that satisfies (国) $C_{1}^{\perp} \subset C_{2}^{\perp}$.

If we fix a rate $R$ lower than $1-\mathrm{H}\left(p_{0}+p_{1}\right)$ and choose a code $C_{2}^{\perp}$ of rate $R$

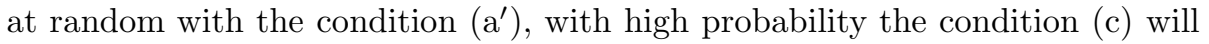
be satisfied [5. Lemma 4]. In this section, we will prove that if we fix a rate $R$ lower than $1-\frac{\mathrm{H}\left(p_{0}\right)+\mathrm{H}\left(p_{1}\right)}{2}$ and choose a code $C_{2}^{\perp}$ of rate $R$ at random with the condition (国), with high probability the condition (드) will be satisfied. Some ideas used in the proof are borrowed from [3, 4].

We present the main theorem in Section 3.1 and the proof of this theorem in Section 3.2 Then we consider the key rate of securely shared key in Section 3.3 and compare our result with Mayers' in Section 3.4

\subsection{The code for privacy amplification}

Given a code $C_{1}^{\perp}$ of dimension $r$, we consider how to choose a code $C_{2}^{\perp}$. Fix a rate $R=\frac{r+m}{n}<1-\frac{\mathrm{H}\left(p_{0}\right)+\mathrm{H}\left(p_{1}\right)}{2}$, and let

$$
\begin{aligned}
A_{m}=\left\{C_{2}^{\perp} \subset \mathbf{F}_{2}^{n} \mid C_{2}^{\perp}\right. \text { is a linear space, } \\
\\
\left.\operatorname{dim} C_{2}^{\perp}=r+m, C_{1}^{\perp} \subset C_{2}^{\perp}\right\}
\end{aligned}
$$

be the set from which we choose a code $C_{2}^{\perp}$. 


\subsubsection{Minimum conditional entropy decoding}

To evaluate the decoding error probability, we employ the minimum conditional entropy decoding [6. Appendix B]. Let $\boldsymbol{e}$ be an error occurred in $n$ bits binary vector, $P_{\zeta}$ be the type of first $\frac{n}{2}$ bits of $\boldsymbol{e}$, and $P_{\eta}$ be the type of latter $\frac{n}{2}$ bits of $\boldsymbol{e}$. Then we define the conditional entropy of $\boldsymbol{e}$ as

$$
\mathrm{H}_{c}(\boldsymbol{e})=\mathrm{H}_{c}\left(P_{\zeta}, P_{\eta}\right)=\frac{\mathrm{H}\left(P_{\zeta}\right)+\mathrm{H}\left(P_{\eta}\right)}{2} .
$$

In the minimum conditional entropy decoding, we find an estimated error $\hat{\boldsymbol{e}}$ that minimizes $\mathrm{H}_{c}(\hat{\boldsymbol{e}})$ and has the same syndrome, i.e., $\hat{\boldsymbol{e}} H_{2}^{T}=e H_{2}^{T}$, where $H_{2}$ is parity check matrix of $C_{2}^{\perp}$.

\subsubsection{The decoding process of a CSS code}

We assume that only phase errors occur because we will consider the decoding process by $C_{2}^{\perp}$. Assume that a codeword $\left|\boldsymbol{v}+C_{2}\right\rangle$ is sent and $\sigma_{z}^{[e]}\left|\boldsymbol{v}+C_{2}\right\rangle$ is received, where

$$
\begin{aligned}
\left|\boldsymbol{v}+C_{2}\right\rangle & =\frac{1}{\left|C_{2}\right|^{1 / 2}} \sum_{\boldsymbol{w} \in C_{2}}|\boldsymbol{v}+\boldsymbol{w}\rangle \quad \boldsymbol{v} \in C_{1}, \\
\sigma_{z}^{[e]} & =\sigma_{z}^{e_{1}} \otimes \cdots \otimes \sigma_{z}^{e_{n}} \\
\boldsymbol{e} & =\left(e_{1}, \cdots, e_{n}\right) .
\end{aligned}
$$

Compute the syndrome $\boldsymbol{e} H_{2}^{T}$ and find an estimated error $\hat{e}$. Then, apply the unitary operator $\sigma_{z}^{[\hat{e}]}$ to $\sigma_{z}^{[e]}\left|\boldsymbol{v}+C_{2}\right\rangle$ to correct the error. If

$$
\sigma_{z}^{[e+\hat{e}]}\left|\boldsymbol{v}+C_{2}\right\rangle \neq\left|\boldsymbol{v}+C_{2}\right\rangle
$$

a decoding error occurs.

\subsubsection{Errors causing decoding errors}

We consider when decoding errors occur. Note that the condition $\hat{e} H_{2}^{T}=e H_{2}^{T}$ is equivalent to $\boldsymbol{e}+\hat{\boldsymbol{e}} \in C_{2}^{\perp}$. For a linear code $C_{2}^{\perp}$, if there exists a vector $\hat{\boldsymbol{e}}$ such that $\boldsymbol{e}+\hat{\boldsymbol{e}} \in C_{2}^{\perp}$ and $\mathrm{H}_{c}(\hat{\boldsymbol{e}}) \leq \mathrm{H}_{c}(\boldsymbol{e})$, the estimated error is $\hat{\boldsymbol{e}}$ instead of $\boldsymbol{e}$. If the unitary operator $\sigma_{z}^{[\boldsymbol{e}+\hat{\boldsymbol{e}}]}$ applied to a codeword of a CSS code $\left|\boldsymbol{v}+C_{2}\right\rangle$, then we have

$$
\begin{aligned}
& \sigma_{z}^{[\boldsymbol{e}+\hat{\boldsymbol{e}}]} \frac{1}{\left|C_{2}\right|^{1 / 2}} \sum_{\boldsymbol{w} \in C_{2}}|\boldsymbol{v}+\boldsymbol{w}\rangle \\
= & \frac{1}{\left|C_{2}\right|^{1 / 2}} \sum_{\boldsymbol{w} \in C_{2}}(-1)^{(\boldsymbol{v}+\boldsymbol{w}) \cdot(\boldsymbol{e}+\hat{\boldsymbol{e}})}|\boldsymbol{v}+\boldsymbol{w}\rangle .
\end{aligned}
$$

If $(\boldsymbol{v}+\boldsymbol{w}) \cdot(\boldsymbol{e}+\hat{\boldsymbol{e}})=0$ for all $\boldsymbol{v}+\boldsymbol{w} \in C_{1}$, the codeword is left unchanged by multiplication of $\sigma_{z}^{[\boldsymbol{e}+\hat{\boldsymbol{e}}]}$. Because $\boldsymbol{v} \in C_{1}, \boldsymbol{w} \in C_{2}$, and $C_{2} \subset C_{1}$, if $\boldsymbol{e}+\hat{\boldsymbol{e}} \in C_{1}^{\perp}$, 
then $(\boldsymbol{v}+\boldsymbol{w}) \cdot(\boldsymbol{e}+\hat{\boldsymbol{e}})=0$. Thus, if $\boldsymbol{e}+\hat{\boldsymbol{e}} \in C_{1}^{\perp}$, the errors are not estimated correctly but the received state will be corrected to the original state, and these errors do not yield decoding errors. In case of decoding a CSS code, we define the set of errors for each $C_{2}^{\perp}$, which cause decoding errors, as

$$
\mathcal{E}\left(C_{2}^{\perp}\right)=\left\{\boldsymbol{e} \in \mathbf{F}_{2}^{n} \mid \exists \hat{\boldsymbol{e}} \mathrm{H}_{c}(\hat{\boldsymbol{e}}) \leq \mathrm{H}_{c}(\boldsymbol{e}), \boldsymbol{e}+\hat{\boldsymbol{e}} \in C_{2}^{\perp} \backslash C_{1}^{\perp}\right\}
$$

Definition 2 We define the decoding error probability of $C_{2}^{\perp}$ as a part of a CSS code over a BSC whose crossover probability of first $\frac{n}{2}$ bits are $p_{1}^{\prime}$ and that of latter $\frac{n}{2}$ bits are $p_{0}^{\prime}$ as

$$
P_{e r r}\left(C_{2}^{\perp}, p_{0}^{\prime}, p_{1}^{\prime}\right)=\sum_{\boldsymbol{e} \in \mathcal{E}\left(C_{2}^{\perp}\right)} Q(\boldsymbol{e})
$$

where $Q(\boldsymbol{e})$ is a probability that $\boldsymbol{e}$ occurs in a BSC whose crossover probability of first $\frac{n}{2}$ bits are $p_{1}^{\prime}$ and that of latter $\frac{n}{2}$ bits are $p_{0}^{\prime}$.

The decoding error probability of $C_{1}$ as a part of a CSS code is defined in the same way considering the decoding process of bit flip errors of a CSS code.

Theorem 3 If we choose a code $C_{2}^{\perp}$ at random from $A_{m}$, for arbitrary $\mu>0$, we have

$$
\begin{array}{r}
\operatorname{Pr}\left\{P_{e r r}\left(C_{2}^{\perp}, p_{0}^{\prime}, p_{1}^{\prime}\right) \leq\left(\frac{n}{2}+1\right)^{4} 2^{-n\left(E\left(R, p_{0}, p_{1}\right)-\mu\right)}\right. \\
\left.\forall p_{0}^{\prime} \leq p_{0}, p_{1}^{\prime} \leq p_{1}\right\} \geq 1-\left(\frac{n}{2}+1\right)^{2} 2^{-\mu n}
\end{array}
$$

where

$$
\begin{aligned}
& E\left(R, p_{0}, p_{1}\right)= \\
& \min _{q_{0}, q_{1}}\left[\frac{D\left(q_{1} \mid p_{1}\right)+D\left(q_{0} \mid p_{0}\right)}{2}+\left|1-R-\mathrm{H}_{c}\left(q_{1}, q_{0}\right)\right|^{+}\right],
\end{aligned}
$$

and $|x|^{+}=\max \{x, 0\}$. Note that $\min _{q_{0}, q_{1}}$ is taken over $0 \leq q_{0}, q_{1} \leq 1$. Because $\frac{D\left(q_{1} \mid p_{1}\right)+D\left(q_{0} \mid p_{0}\right)}{2}=0$ only if $q_{1}=p_{1}, q_{0}=p_{0}$, and $R<1-\frac{\mathrm{H}\left(p_{0}\right)+\mathrm{H}\left(p_{1}\right)}{2}$, we have $E\left(R, p_{0}, p_{1}\right)>0$.

Consequently, we can obtain a code $C_{2}^{\perp}$ that satisfy the condition (ㄷ) with high probability by choosing a code at random from $A_{m}$.

\subsection{Proof of the theorem}

Refer to 9 for the method of type used in this section. We also use the notation $T_{\left(P_{\zeta}, P_{\eta}\right)}^{n}$ as the set of binary vectors whose type of first $\frac{n}{2}$ bits is $P_{\zeta}$ and that of latter $\frac{n}{2}$ bits is $P_{\eta}$. $P_{\frac{n}{2}}^{2}$ is the direct product of the sets of all possible types over $\{0,1\}^{\frac{n}{2}}$, i.e., $P_{\frac{n}{2}} \times P_{\frac{n}{2}}$. 
We classify $\mathcal{E}\left(C_{2}^{\perp}\right)$ by the types in $P_{\frac{n}{2}}^{2}$ as

$$
\mathcal{E}\left(C_{2}^{\perp}\right)=\bigcup_{\left(P_{\zeta}, P_{\eta}\right)} \mathcal{E}_{\left(P_{\zeta}, P_{\eta}\right)}\left(C_{2}^{\perp}\right)
$$

where $\mathcal{E}_{\left(P_{\zeta}, P_{\eta}\right)}\left(C_{2}^{\perp}\right)=\mathcal{E}\left(C_{2}^{\perp}\right) \cap T_{\left(P_{\zeta}, P_{\eta}\right)}^{n}$. First, we prove that if we choose a code $C_{2}^{\perp}$ at random from $A_{m}, C_{2}^{\perp}$ satisfies the following property with high probability. Then we prove that the decoding error probability of $C_{2}^{\perp}$ that satisfy the following property is small. Given arbitrary $\mu>0$, for all types $\left(P_{\zeta}, P_{\eta}\right) \in P_{\frac{n}{2}}^{2}$,

$$
\frac{\left|\mathcal{E}_{\left(P_{\zeta}, P_{\eta}\right)}\left(C_{2}^{\perp}\right)\right|}{\left|T_{\left(P_{\zeta}, P_{\eta}\right)}^{n}\right|} \leq 2^{-n\left(\left|1-\mathrm{H}_{c}\left(P_{\zeta}, P_{\eta}\right)-R\right|^{+}-\mu\right)} .
$$

To prove this, we evaluate the average of $\frac{\left|\mathcal{E}_{\left(P_{\zeta}, P_{\eta}\right)}\left(C_{2}^{\perp}\right)\right|}{\left|T_{\left(P_{\zeta}, P_{\eta}\right)}^{n}\right|}$ over $C_{2}^{\perp} \in A_{m}$. Define the set of codes that cannot correct $e$ as

$$
B_{m}(\boldsymbol{e})=\left\{C_{2}^{\perp} \in A_{m} \mid \boldsymbol{e} \in \mathcal{E}\left(C_{2}^{\perp}\right)\right\} .
$$

Define $C_{m}(\boldsymbol{e})$ as

$$
C_{m}(\boldsymbol{e})=\left\{C_{2}^{\perp} \in A_{m} \mid \boldsymbol{e} \in C_{2}^{\perp} \backslash C_{1}^{\perp}\right\}
$$

and $G$ as the set of bijective linear maps $\alpha$ on $\mathbf{F}_{2}^{n}$ that satisfies $\alpha\left(C_{1}^{\perp}\right)=C_{1}^{\perp}$. Then we have the following equalities:

$$
\begin{aligned}
& \left|C_{m}(\boldsymbol{e})\right| \\
= & \left|\left\{C_{2}^{\perp} \in A_{m} \mid \boldsymbol{e} \in C_{2}^{\perp} \backslash C_{1}^{\perp}\right\}\right| \\
= & \mid\left\{\alpha\left(C_{2}^{\perp}\right) \mid \boldsymbol{e} \in \alpha\left(C_{2}^{\perp} \backslash C_{1}^{\perp}\right), \alpha \in G, C_{2}^{\perp} \text { is fixed }\right\} \mid \\
= & \mid\left\{\beta \alpha\left(C_{2}^{\perp}\right) \mid \beta(\boldsymbol{e}) \in \beta \alpha\left(C_{2}^{\perp} \backslash C_{1}^{\perp}\right), \alpha, \beta \in G,\right. \\
& \left.\quad \beta \text { and } C_{2}^{\perp} \text { are fixed }\right\} \mid .
\end{aligned}
$$

Since there exists $\beta \in G$ such that $\boldsymbol{e}^{\prime}=\beta(\boldsymbol{e})$ for arbitrary $\boldsymbol{e}$ and $\boldsymbol{e}^{\prime} \in \mathbf{F}_{2}^{n} \backslash C_{1}^{\perp}$, $\left|C_{m}(\boldsymbol{e})\right|$ does not depend on $\boldsymbol{e} \in \mathbf{F}_{2}^{n} \backslash C_{1}^{\perp}$ and

$$
\begin{aligned}
\left|C_{m}(\boldsymbol{e})\right| & =\frac{\sum_{\boldsymbol{e} \in \mathbf{F}_{2}^{n} \backslash C_{1}^{\perp}}\left|C_{m}(\boldsymbol{e})\right|}{\left|\mathbf{F}_{2}^{n} \backslash C_{1}^{\perp}\right|} \\
& =\frac{\sum_{\boldsymbol{e} \in \mathbf{F}_{2}^{n} \backslash C_{1}^{\perp}}\left|\left\{C_{2}^{\perp} \in A_{m} \mid \boldsymbol{e} \in C_{2}^{\perp} \backslash C_{1}^{\perp}\right\}\right|}{\left|\mathbf{F}_{2}^{n} \backslash C_{1}^{\perp}\right|} \\
& =\frac{\sum_{C_{2}^{\perp} \in A_{m}}\left|\left\{\boldsymbol{e} \in \mathbf{F}_{2}^{n} \backslash C_{1}^{\perp} \mid \boldsymbol{e} \in C_{2}^{\perp} \backslash C_{1}^{\perp}\right\}\right|}{\left|\mathbf{F}_{2}^{n} \backslash C_{1}^{\perp}\right|} \\
& =\frac{\left|C_{2}^{\perp} \backslash C_{1}^{\perp}\right|\left|A_{m}\right|}{\left|\mathbf{F}_{2}^{n} \backslash C_{1}^{\perp}\right|} .
\end{aligned}
$$


From the definition, it is obvious that $\left|C_{m}(\boldsymbol{e})\right|=0$ for $\boldsymbol{e} \in C_{1}^{\perp}$. Hence

$$
\begin{aligned}
\left|C_{m}(\boldsymbol{e})\right| & \leq \frac{\left|C_{2}^{\perp} \backslash C_{1}^{\perp}\right|\left|A_{m}\right|}{\left|\mathbf{F}_{2}^{n} \backslash C_{1}^{\perp}\right|} \\
& =\frac{2^{r+m}-2^{r}}{2^{n}-2^{r}}\left|A_{m}\right| \\
& =\frac{\left|A_{m}\right|}{2^{n-(r+m)}} \frac{1-2^{-m}}{1-2^{-n+r}} \\
& \leq \frac{\left|A_{m}\right|}{2^{n-(r+m)}} \\
& =\left|A_{m}\right| 2^{-n(1-R)} .
\end{aligned}
$$

Because the condition for $C_{2}^{\perp} \in A_{m}$ to belong to $B_{m}(\boldsymbol{e})$ is $\exists \hat{\boldsymbol{e}} \mathrm{H}_{c}(\hat{\boldsymbol{e}}) \leq$ $\mathrm{H}_{c}(\boldsymbol{e}), \boldsymbol{e}+\hat{\boldsymbol{e}} \in C_{2}^{\perp} \backslash C_{1}^{\perp}$, we obtain

$$
\begin{aligned}
\frac{\left|B_{m}(e)\right|}{\left|A_{m}\right|} & \leq \frac{1}{\left|A_{m}\right|} \sum_{\substack{\hat{e} \in \mathbf{F}_{2}^{n} \\
\mathrm{H}_{c}(\hat{\boldsymbol{e}}) \leq \mathrm{H}_{c}(\boldsymbol{e})}}\left|C_{m}(\boldsymbol{e}+\hat{\boldsymbol{e}})\right| \\
& \leq \sum_{\substack{\hat{\boldsymbol{e}} \in \mathbf{F}_{2}^{n} \\
\mathrm{H}_{c}(\hat{\boldsymbol{e}}) \leq \mathrm{H}_{c}(\boldsymbol{e})}} 2^{-n(1-R)},
\end{aligned}
$$

while $\frac{\left|B_{m}(e)\right|}{\left|A_{m}\right|} \leq 1$. Let $|x|^{+}=\max \{x, 0\}$ and note that if $a, b \geq 0$, then $\min \{a+$ $b, 1\} \leq \min \{a, 1\}+\min \{b, 1\}$.

Using above definitions, we have

$$
\begin{aligned}
& \frac{1}{\left|A_{m}\right|} \sum_{C_{2}^{\perp} \in A_{m}} \frac{\left|\mathcal{E}_{\left(P_{\zeta}, P_{\eta}\right)}\left(C_{2}^{\perp}\right)\right|}{\left|T_{\left(P_{\zeta}, P_{\eta}\right)}^{n}\right|} \\
& =\frac{1}{\left|T_{\left(P_{\zeta}, P_{\eta}\right)}^{n}\right|} \sum_{\boldsymbol{e} \in T_{\left(P_{\zeta}, P_{\eta}\right)}^{n}} \frac{\left|B_{m}(\boldsymbol{e})\right|}{\left|A_{m}\right|} \\
& \leq \frac{1}{\left|T_{\left(P_{\zeta}, P_{\eta}\right)}^{n}\right|} \sum_{\boldsymbol{e} \in T_{\left(P_{\zeta}, P_{\eta}\right)}^{n}} \min \left\{\sum_{\substack{\hat{e} \in \mathbf{F}_{2}^{n} \\
\mathrm{H}_{c}(\hat{\boldsymbol{e}}) \leq \mathrm{H}_{c}(\boldsymbol{e})}} 2^{-n(1-R)}, 1\right\} \\
& =\min \left\{\sum_{\substack{\left(P_{\zeta}^{\prime}, P_{\eta}^{\prime}\right) \in P_{\frac{n}{2}}^{2} \\
\mathrm{H}_{c}\left(P_{\zeta}^{\prime}, P_{\eta}^{\prime}\right) \leq \mathrm{H}_{c}\left(P_{\zeta}, P_{\eta}\right)}}\left|T_{\left(P_{\zeta}^{\prime}, P_{\eta}^{\prime}\right)}^{n}\right| 2^{-n(1-R)}, 1\right\} \\
& \leq \sum_{\substack{\left(P_{\zeta}^{\prime}, P_{\eta}^{\prime}\right) \in P_{\frac{n}{2}}^{2} \\
\mathrm{H}_{c}\left(P_{\zeta}^{\prime}, P_{\eta}^{\prime}\right) \leq \mathrm{H}_{c}\left(P_{\zeta}, P_{\eta}\right)}} 2^{-n\left|1-R-\mathrm{H}_{c}\left(P_{\zeta}^{\prime}, P_{\eta}^{\prime}\right)\right|^{+}}
\end{aligned}
$$




$$
\begin{aligned}
& \leq\left|P_{\frac{n}{2}}^{2}\right| \max _{\substack{\left(P_{\zeta}^{\prime}, P_{\eta}^{\prime}\right) \in P_{\frac{n}{2}}^{2} \\
\mathrm{H}_{c}\left(P_{\zeta}^{\prime}, P_{\eta}^{\prime}\right) \leq \mathrm{H}_{c}\left(P_{\zeta}, P_{\eta}\right)}} 2^{-n\left|1-R-\mathrm{H}_{c}\left(P_{\zeta}^{\prime}, P_{\eta}^{\prime}\right)\right|^{+}} \\
& \leq\left(\frac{n}{2}+1\right)^{2} 2^{-n\left|1-R-\mathrm{H}_{c}\left(P_{\zeta}, P_{\eta}\right)\right|^{+}}
\end{aligned}
$$

Let $A_{b}\left(\mu, P_{\zeta}, P_{\eta}\right)$ and $A_{g}(\mu)$ be

$$
\begin{aligned}
A_{b}\left(\mu, P_{\zeta}, P_{\eta}\right)= & \left\{C_{2}^{\perp} \in A_{m} \mid \frac{\left|\mathcal{E}_{\left(P_{\zeta}, P_{\eta}\right)}\left(C_{2}^{\perp}\right)\right|}{\left|T_{\left(P_{\zeta}, P_{\eta}\right)}^{n}\right|}\right. \\
> & \left.\left(\frac{n}{2}+1\right)^{2} 2^{-n\left(\left|1-R-\mathrm{H}_{c}\left(P_{\zeta}, P_{\eta}\right)\right|^{+}-\mu\right)}\right\}, \\
A_{g}(\mu)=A_{m} \backslash & \bigcup_{\left(P_{\zeta}, P_{\eta}\right) \in P_{\frac{n}{2}}^{2}} A_{b}\left(\mu, P_{\zeta}, P_{\eta}\right) .
\end{aligned}
$$

From the union bound and the Chebychev inequality, we have

$$
\begin{aligned}
& \frac{\left|A_{g}(\mu)\right|}{\left|A_{m}\right|} \\
= & 1-\frac{\bigcup_{\left(P_{\zeta}, P_{\eta}\right) \in P_{\frac{n}{2}}^{2}}\left|A_{g}\left(\mu, P_{\zeta}, P_{\eta}\right)\right|}{\left|A_{m}\right|} \\
\geq & 1-\sum_{\left(P_{\zeta}, P_{\eta}\right) \in P_{\frac{n}{2}}^{2}} \frac{\left|A_{g}\left(\mu, P_{\zeta}, P_{\eta}\right)\right|}{\left|A_{m}\right|} \\
\geq & 1-\sum_{\left(P_{\zeta}, P_{\eta}\right) \in P_{\frac{n}{2}}^{2}} \frac{\left(\frac{n}{2}+1\right)^{2} 2^{-n\left|1-R-\mathrm{H}_{c}\left(P_{\zeta}, P_{\eta}\right)\right|^{+}}}{\left(\frac{n}{2}+1\right)^{2} 2^{-n\left(\left|1-R-\mathrm{H}_{c}\left(P_{\zeta}, P_{\eta}\right)\right|^{+}-\mu\right)}} \\
\geq & 1-\left(\frac{n}{2}+1\right)^{2} 2^{-\mu n}
\end{aligned}
$$

Next, we evaluate the decoding error probability of $C_{2}^{\perp} \in A_{g}(\mu)$. Let $p_{0}^{\prime} \leq p_{0}$ and $p_{1}^{\prime} \leq p_{1}$, and $Q(\boldsymbol{e})$ be a probability that $\boldsymbol{e}$ occurs in a BSC whose crossover probability of first $\frac{n}{2}$ bits are $p_{1}^{\prime}$ and that of latter $\frac{n}{2}$ bits are $p_{0}^{\prime}$. Then the decoding error probability of $C_{2}^{\perp}$ as a part of a CSS code is

$$
\begin{aligned}
& =\sum_{\boldsymbol{e} \in \mathcal{E}\left(C_{2}^{\perp}\right)}^{P_{e r r}\left(C_{2}^{\perp}, p_{0}^{\prime}, p_{1}^{\prime}\right)} Q(\boldsymbol{e}) \\
= & \sum_{\left(P_{\zeta}, P_{\eta}\right) \in P_{\frac{n}{2}}^{2}} \sum_{\boldsymbol{e} \in \mathcal{E}_{\left(P_{\zeta}, P_{\eta}\right)}\left(C_{2}^{\perp}\right)} Q(\boldsymbol{e}) \\
= & \sum_{\left(P_{\zeta}, P_{\eta}\right) \in P_{\frac{n}{2}}^{2}} \frac{\left|\mathcal{E}_{\left(P_{\zeta}, P_{\eta}\right)}\left(C_{2}^{\perp}\right)\right|}{\left|T_{\left(P_{\zeta}, P_{\eta}\right)}^{n}\right|} Q\left(T_{\left(P_{\zeta}, P_{\eta}\right)}^{n}\right)
\end{aligned}
$$




$$
\begin{aligned}
\leq & \sum_{\left(P_{\zeta}, P_{\eta}\right) \in P_{\frac{n}{2}}^{2}}\left(\frac{n}{2}+1\right)^{2} 2^{-n\left(\left|1-R-\mathrm{H}_{c}\left(P_{\zeta}, P_{\eta}\right)\right|^{+}-\mu\right)} \\
& \times 2^{-\frac{n}{2}\left\{D\left(P_{\zeta}(1) \mid p_{1}^{\prime}\right)+D\left(P_{\eta}(1) \mid p_{0}^{\prime}\right)\right\}} \\
\leq & \left(\frac{n}{2}+1\right)^{4} 2^{-n\left(E\left(R, p_{0}^{\prime}, p_{1}^{\prime}\right)-\mu\right)}
\end{aligned}
$$

where

$$
\begin{aligned}
& E\left(R, p_{0}^{\prime}, p_{1}^{\prime}\right)= \\
& \min _{q_{0}, q_{1}}\left[\frac{D\left(q_{1} \mid p_{1}^{\prime}\right)+D\left(q_{0} \mid p_{0}^{\prime}\right)}{2}+\left|1-R-\mathrm{H}_{c}\left(q_{1}, q_{0}\right)\right|^{+}\right] .
\end{aligned}
$$

\section{Lemma 4}

$$
\min _{\substack{0 \leq p_{0}^{\prime} \leq p_{0} \\ 0 \leq p_{1}^{\prime} \leq p_{1}}} E\left(R, p_{0}^{\prime}, p_{1}^{\prime}\right)=E\left(R, p_{0}, p_{1}\right)
$$

We prove this lemma in Appendix $\mathbb{B}$ From this lemma, we have

$$
\begin{aligned}
& P_{\text {err }}\left(C_{2}^{\perp}, p_{0}^{\prime}, p_{1}^{\prime}\right) \\
\leq \quad & \left(\frac{n}{2}+1\right)^{4} 2^{-n\left(E\left(R, p_{0}, p_{1}\right)-\mu\right)} \quad \forall p_{0}^{\prime} \leq p_{0}, p_{1}^{\prime} \leq p_{1} .
\end{aligned}
$$

Then Theorem 3 is proved.

\subsection{Achievable key rate}

We proved that if we fix a code $C_{1}^{\perp}$ and choose a code $C_{2}^{\perp}$ of a fixed rate $R<1-\frac{\mathrm{H}\left(p_{0}\right)+\mathrm{H}\left(p_{1}\right)}{2}$ at random with the condition $C_{1}^{\perp} \subset C_{2}^{\perp}$, the decoding error probability of $C_{2}^{\perp}$ as a part of a CSS code is small with high probability. Consequently, we can conduct random privacy amplification with a code $C_{2}$ of a rate higher than $\frac{\mathrm{H}\left(p_{0}\right)+\mathrm{H}\left(p_{1}\right)}{2}$.

If we estimate an error rate in a lump (test bits in each basis are lumped together and a single error rate is computed), an estimated error rate is $\frac{p_{0}+p_{1}}{2}$ instead of $p_{0}$ and $p_{1}$. Thus, we can conduct random privacy amplification with a code $C_{2}$ with a rate higher than $\mathrm{H}\left(\frac{p_{0}+p_{1}}{2}\right)$. Since the entropy function is concave, we can conduct random privacy amplification with a code $C_{2}$ of a lower rate by estimating error rates separately, which enables us to share a longer key.

\subsection{Comparison with Mayers' evaluation}

In this section, we compare our result with Mayers'. In case of estimating an error rate in a lump, we can conduct random privacy amplification with a code $C_{2}$ of a rate higher than $\mathrm{H}\left(\frac{p_{0}+p_{1}}{2}\right)$. Because there exists efficiently decodeable codes whose rate is fairly close to $1-\mathrm{H}\left(\frac{p_{0}+p_{1}}{2}\right)$ and whose decoding error 


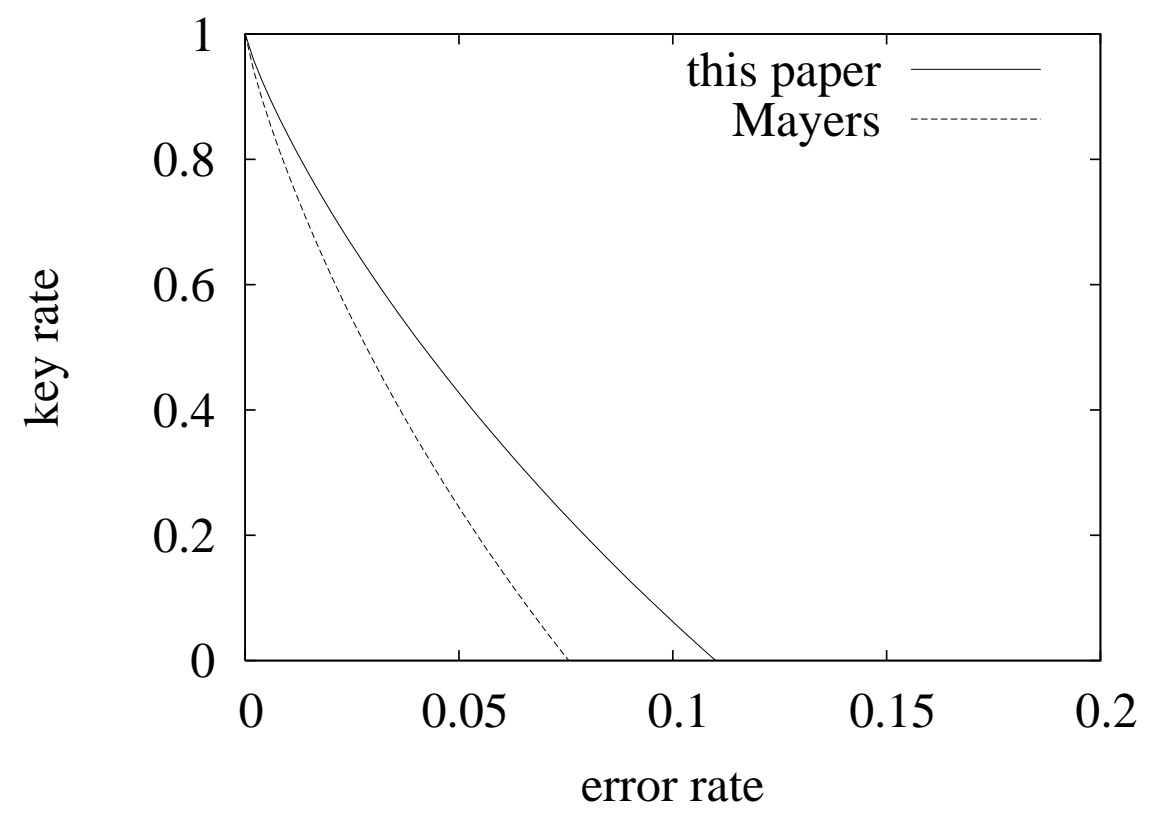

Figure 1: Comparison of achievable key rates.

probability is small, we can securely share a key with a rate lower than 1 $2 \mathrm{H}\left(\frac{p_{0}+p_{1}}{2}\right)$. With Mayers' evaluation of minimum Hamming weight of $C_{2}^{\perp} \backslash C_{1}^{\perp}$ in [5], we can securely share a key with a rate lower than $1-\mathrm{H}\left(\frac{p_{0}+p_{1}}{2}\right)-\mathrm{H}\left(p_{0}+\right.$ $\left.p_{1}\right)$, where $1-\mathrm{H}\left(\frac{p_{0}+p_{1}}{2}\right)$ is the rate of $C_{1}$ for error correction and $\mathrm{H}\left(p_{0}+p_{1}\right)$ is the rate of $C_{2}$ for privacy amplification.

According to the evaluation of the decoding error probability, we showed that the tolerable error rate can be increased from $7.5 \%$ to $11 \%$ in the BB84 protocol with random privacy amplification. Figure 1 shows the secure key rate of this paper and Mayers' against error rate, and the key rate falls to 0 at the point of $7.5 \%$ and $11 \%$ respectively.

\section{Conclusion}

For a fixed code $C_{1}$, we showed that we can decrease a rate of randomly chosen code $C_{2}$, keeping the BB84 protocol to be secure. Consequently, we proved that the $\mathrm{BB} 84$ protocol with random privacy amplification can tolerate severer noise and can share longer keys. 


\section{Acknowledgment}

We appreciate the helpful comment by Dr. Masahito Hayashi on an earlier version of this paper. This research is partly supported by the Japan Society for the Promotion of Science.

\section{A Proof of theorem 1}

In this appendix, we prove Theorem 1 i.e., we prove that we can securely share a key against general eavesdropping attacks with the linear codes $C_{1}$ and $C_{2}$ that satisfy the conditions (ㅈad)-(ㄷ) in Section 2.2. Basic ideas in this proof are borrowed from [6, 7].

The outline of this proof is similar to the proof in $[6]$. We introduce a class of CSS codes and the CSS code protocol in Sections A.1 and A.2 respectively. Then we define some notations used in this proof in Section A.3. After that, we define Eve's attack mathematically and relate the BB84 protocol to the CSS code protocol in Sections A.4 and A.5 respectively. In Section A.6 in order to bound the mutual information, we bound the fidelity, which is defined in Section A.5. Finally, we upper bound the mutual information of a shared key and Eve's accessible information in Section A.7 using the result in Section A.6

\section{A.1 A class of CSS codes}

In this section, we define a class of CSS codes. A CSS code $\mathcal{Q}$ is constructed from two linear codes $C_{1}$ and $C_{2}$ that satisfy $C_{2} \subset C_{1}$. A codeword $\left|\phi_{\boldsymbol{u}}\right\rangle \in \mathcal{Q}$ is

$$
\left|\phi_{\boldsymbol{u}}\right\rangle=\frac{1}{\sqrt{\left|C_{2}\right|}} \sum_{\boldsymbol{w} \in C_{2}}|\boldsymbol{u}+\boldsymbol{w}\rangle,
$$

where $\boldsymbol{u}$ is a coset representative of $C_{1} / C_{2}$. A class of CSS codes is $\left\{\mathcal{Q}_{\boldsymbol{x} z}\right\}$ that are parametrized by coset representatives $\boldsymbol{x} \in \mathbf{F}_{2}^{n} / C_{1}$ and $\boldsymbol{z} \in \mathbf{F}_{2}^{n} / C_{2}^{\perp}$, and a codeword $\left|\phi_{\boldsymbol{u x} \boldsymbol{z}}\right\rangle \in \mathcal{Q}_{\boldsymbol{x} \boldsymbol{z}}$ is

$$
\left|\phi_{\boldsymbol{u x} \boldsymbol{z}}\right\rangle=\frac{1}{\sqrt{\left|C_{2}\right|}} \sum_{\boldsymbol{w} \in C_{2}}(-1)^{\boldsymbol{z} \cdot \boldsymbol{w}}|\boldsymbol{u}+\boldsymbol{w}+\boldsymbol{x}\rangle
$$

A recovery operator for a CSS code $\mathcal{Q}_{\boldsymbol{x} z}$ is a TPCP (Trace Preserving Completely Positive) map on $\mathrm{L}\left(\mathcal{H}^{\otimes n}\right)$ that represent the measurement of syndrome and the unitary operation of error correction, where $\mathcal{H}$ is a Hilbert space of dimension 2 , and $\mathrm{L}(\mathcal{H})$ is the linear space of operators on $\mathcal{H}$. We denote by $\mathcal{R}_{\boldsymbol{x} z}$ the recovery operator for $\mathcal{Q}_{\boldsymbol{x} \boldsymbol{z}}$.

\section{A.2 The CSS code protocol}

To prove the security of the BB84 protocol, we relate the BB84 protocol with the following CSS code protocol [6] Section 4]. In this section, we use bold large 
letters for random variables and small bold letters for their realizations, e.g., $\boldsymbol{x}$ denote a realization of a random variable $\boldsymbol{X}$. We also use a notation $\mathbb{E}_{\boldsymbol{x}}$ as the expectation operator over $\boldsymbol{X}$.

Suppose Alice chooses coset representatives $\boldsymbol{u} \in C_{1} / C_{2}, \boldsymbol{x} \in \mathbf{F}_{2}^{n} / C_{1}, \boldsymbol{z} \in$ $\mathbf{F}_{2}^{n} / C_{2}^{\perp}$ at random and send $\left|\phi_{\boldsymbol{u x} \boldsymbol{z}}\right\rangle$. Let $\mathcal{A}_{n}$ be a TPCP map on $\mathrm{L}\left(\mathcal{H}^{\otimes n}\right)$ representing Eve's eavesdropping attack, and $\boldsymbol{E}$ be a random variable that is Eve's measurement result. According to [12, Section 5.3], we can bound the mutual information between $\boldsymbol{U}$ and $\boldsymbol{E}$ as

$$
\mathrm{I}(\boldsymbol{U} ; \boldsymbol{E} \mid \boldsymbol{X}=\boldsymbol{x}, \boldsymbol{Z}=\boldsymbol{z}) \leq S_{\boldsymbol{x} \boldsymbol{z}},
$$

where $S_{\boldsymbol{x} \boldsymbol{z}}$ is the entropy exchange [12, Section 5] after the system suffers a Eve's attack $\mathcal{A}_{n}$ and recovery operator $\mathcal{R}_{\boldsymbol{x} \boldsymbol{z}}$, i.e.,

$$
\begin{aligned}
S_{\boldsymbol{x} \boldsymbol{z}} & =S(\rho), \\
\rho & =\left[\mathcal{I}_{n} \otimes \mathcal{R}_{\boldsymbol{x} \boldsymbol{z}}\right]\left(\left[\mathcal{I}_{n} \otimes \mathcal{A}_{n}\right]\left(\left|\Phi_{\boldsymbol{x} \boldsymbol{z}}\right\rangle\left\langle\Phi_{\boldsymbol{x} \boldsymbol{z}}\right|\right)\right), \\
\left|\Phi_{\boldsymbol{x} \boldsymbol{z}}\right\rangle & =\frac{1}{\sqrt{\left|C_{1} / C_{2}\right|}} \sum_{\boldsymbol{u} \in C_{1} / C_{2}}\left|\phi_{\boldsymbol{u} \boldsymbol{x} \boldsymbol{z}}\right\rangle \otimes\left|\phi_{\boldsymbol{u} \boldsymbol{x} \boldsymbol{z}}\right\rangle,
\end{aligned}
$$

and $S(\cdot)$ denotes the von Neumann entropy. Let $F_{\boldsymbol{x} \boldsymbol{z}}$ be the entanglement fidelity of above process, i.e.,

$$
F_{\boldsymbol{x} \boldsymbol{z}}=\left\langle\Phi_{\boldsymbol{x} \boldsymbol{z}}|\rho| \Phi_{\boldsymbol{x} \boldsymbol{z}}\right\rangle .
$$

Then, by the quantum Fano inequality, Eq. (24) of [12, Section 6.2], $S_{\boldsymbol{x} \boldsymbol{z}}$ is bounded as

$$
S_{\boldsymbol{x} \boldsymbol{z}} \leq \mathrm{H}\left(1-F_{\boldsymbol{x} \boldsymbol{z}}\right)+\left(1-F_{\boldsymbol{x} \boldsymbol{z}}\right) 2 n,
$$

where $\mathrm{H}(\cdot)$ is the binary entropy function. Combining Eqs. (11) and (2) and taking the average of the both sides over $\boldsymbol{x}, \boldsymbol{z}$, we have

$$
\mathrm{I}(\boldsymbol{U} ; \boldsymbol{E} \mid \boldsymbol{X}, \boldsymbol{Z}) \leq \mathrm{H}\left(1-\mathbb{E}_{\boldsymbol{x} \boldsymbol{z}} F_{\boldsymbol{x} \boldsymbol{z}}\right)+\left(1-\mathbb{E}_{\boldsymbol{x} \boldsymbol{z}} F_{\boldsymbol{x} \boldsymbol{z}}\right) 2 n,
$$

where we used concavity of entropy function, i.e., $\mathbb{E}_{\boldsymbol{x} \boldsymbol{z}} \mathrm{H}\left(1-F_{\boldsymbol{x} \boldsymbol{z}}\right) \leq \mathrm{H}(1-$ $\mathbb{E}_{\boldsymbol{x} \boldsymbol{z}} F_{\boldsymbol{x} \boldsymbol{z}}$ ). From Eq. (27) of [6] Section 5], the entanglement fidelity $F_{\boldsymbol{x} \boldsymbol{z}}$ is bounded as

$$
1-\mathbb{E}_{\boldsymbol{x} \boldsymbol{z}} F_{\boldsymbol{x} \boldsymbol{z}} \leq \sum_{\left(\boldsymbol{e}_{x}, \boldsymbol{e}_{z}\right) \in \mathcal{E}} \mathcal{P}\left(\boldsymbol{e}_{x}, \boldsymbol{e}_{z}\right),
$$

where $\mathcal{E}$ is the set of uncorrectable errors of a CSS code $\mathcal{Q}$,

$$
\begin{aligned}
\mathcal{P}\left(\boldsymbol{e}_{x}, \boldsymbol{e}_{z}\right) & =\left\langle\Psi_{\boldsymbol{e}_{x} \boldsymbol{e}_{z}}^{n}\left|\left[\mathcal{I}_{n} \otimes \mathcal{A}_{n}\right]\left(\left|\Psi^{n}\right\rangle\left\langle\Psi^{n}\right|\right)\right| \Psi_{\boldsymbol{e}_{x} \boldsymbol{e}_{z}}^{n}\right\rangle, \\
\left|\Psi^{n}\right\rangle & =\frac{1}{\sqrt{2^{n}}} \sum_{\boldsymbol{l} \in \mathbf{F}_{2}^{n}}|\boldsymbol{l}\rangle \otimes|\boldsymbol{l}\rangle, \\
\left|\Psi_{\boldsymbol{e}_{x} \boldsymbol{e}_{z}}^{n}\right\rangle & =\frac{1}{\sqrt{2^{n}}} \sum_{\boldsymbol{l} \in \mathbf{F}_{2}^{n}}|\boldsymbol{l}\rangle \otimes \sigma_{x}^{\left[\boldsymbol{e}_{x}\right]} \sigma_{z}^{\left[\boldsymbol{e}_{z}\right]}|\boldsymbol{l}\rangle .
\end{aligned}
$$




\section{A.3 Notation}

First, we fix the positions of remaining $2 n$ bits out of $(4+\theta) n$ bits in step (9), and represent these positions by $T$. Lets $\boldsymbol{a}_{T}, \boldsymbol{b}_{T} \in \mathbf{F}_{2}^{2 n}$ be the subsequences of $\boldsymbol{a}, \boldsymbol{b}$ that correspond to $T$, which include $n$ bits where $a_{i}=b_{i}=0$ and $n$ bits where $a_{i}=b_{i}=1$. We further divide the positions $T$ into four blocks, $T_{0}^{k}, T_{0}^{t}, T_{1}^{k}, T_{1}^{t}$. $T_{0}^{k}, T_{0}^{t}$ consist of the positions that $a_{i}=b_{i}=0$, and $T_{1}^{k}, T_{1}^{t}$ consist of the positions that $a_{i}=b_{i}=1 . T_{0}^{k}, T_{1}^{k}$ are the positions used for generating a key, and $T_{0}^{t}, T_{1}^{t}$ are the positions used for estimating an error rate. $T_{0}^{k}, T_{0}^{t}, T_{1}^{k}, T_{1}^{t}$ depend on $\boldsymbol{a}_{T}, \boldsymbol{b}_{T}, \boldsymbol{p o s}$, where $\boldsymbol{p o s}$ represents how to divide the remaining positions $T$ into the positions $T^{k}$ for generating a key and the positions $T^{t}$ for estimating an error rate. Note that $T^{k}$ consists of $T_{0}^{k}$ and $T_{1}^{k}$, and $T^{t}$ consists of $T_{0}^{t}$ and $T_{1}^{t}$.

We also use a notation $\boldsymbol{k}_{T}$ as a subsequence of $\boldsymbol{k} \in \mathbf{F}_{2}^{(4+\theta) n}$ that corresponds to $T$, and $\boldsymbol{k}_{T_{0}^{k}}, \boldsymbol{k}_{T_{1}^{k}}, \boldsymbol{k}_{T_{0}^{t}}, \boldsymbol{k}_{T_{1}^{t}} \boldsymbol{k}_{T^{k}}, \boldsymbol{k}_{T^{t}}$ are subsequences of $\boldsymbol{k}_{T}$ that corresponds to $T_{0}^{k}, T_{1}^{k}, T_{0}^{t}, T_{1}^{t}, T^{k}, T^{t}$ respectively. Subsequences of $\boldsymbol{c}, \boldsymbol{d}, \tilde{\boldsymbol{k}} \in \mathbf{F}_{2}^{(4+\theta) n}$ are defined in the same way.

\section{A.4 Eve's attack}

Let a TPCP map $\mathcal{A}: \mathrm{L}\left(\mathcal{H}^{\otimes(4+\theta) n}\right) \rightarrow \mathrm{L}\left(\mathcal{H}^{\otimes(4+\theta) n}\right)$ represent Eve's eavesdropping attack (plus channel noise) on transmitted $(4+\theta) n$ qubits. Note that $\mathcal{A}$ does not depend on $\boldsymbol{a}, \boldsymbol{b}, \boldsymbol{p o s}, \pi$. In the BB84 protocol, Alice chooses $(4+\theta) n$ bit string $\boldsymbol{k}$ and sends it with either $\{|0\rangle,|1\rangle\}$ or $\{|+\rangle,|-\rangle\}$ basis according to $\boldsymbol{a}$, i.e., Alice sends $H^{[\boldsymbol{a}]}|\boldsymbol{k}\rangle$, where $H^{[\boldsymbol{a}]}=H^{a_{1}} \otimes \cdots \otimes H^{a_{(4+\theta)}}$, and $H$ is a Hadamard transformation. Bob receives $\mathcal{A}\left(H^{[\boldsymbol{a}]}|\boldsymbol{k}\rangle\langle\boldsymbol{k}| H^{[\boldsymbol{a}]}\right)$, and measures it by either $\{|0\rangle,|1\rangle\}$ or $\{|+\rangle,|-\rangle\}$ basis according to $\boldsymbol{b}$ and obtain $\tilde{\boldsymbol{k}}$. Note that $[H \otimes H]|\Psi\rangle=|\Psi\rangle$, where

$$
|\Psi\rangle=\frac{|0\rangle|0\rangle+|1\rangle|1\rangle}{\sqrt{2}},
$$

and that we can denote Eve's attack by a unitary operator on Bob's system and Eve's system $\mathcal{H}^{\otimes(4+\theta) n} \otimes \mathcal{H}_{E}$, i.e.,

$$
U_{B E}\left[H^{[a]}|\boldsymbol{k}\rangle \otimes\left|e_{0}\right\rangle\right],
$$

where $|e\rangle_{0}$ is a state in $\mathcal{H}_{E}$, and $U_{B E}$ is an unitary operation on $\mathcal{H}^{(4+\theta) n} \otimes \mathcal{H}_{E}$. We can mathematically regard Alice's sent bits $\boldsymbol{k}$ and Bob's received bits $\tilde{\boldsymbol{k}}$ as follows. First, Alice and Bob share a bipartite state

$$
\begin{aligned}
\left|\Psi^{(4+\theta) n}\right\rangle & =\frac{1}{\sqrt{2^{(4+\theta) n}}} \sum_{\boldsymbol{l} \in \mathbf{F}_{2}^{(4+\theta) n}} H^{[\boldsymbol{a}]}|\boldsymbol{l}\rangle \otimes H^{[\boldsymbol{a}]}|\boldsymbol{l}\rangle \\
& =\frac{1}{\sqrt{2^{(4+\theta) n}}} \sum_{\boldsymbol{l} \in \mathbf{F}_{2}^{(4+\theta) n}}|\boldsymbol{l}\rangle \otimes|\boldsymbol{l}\rangle
\end{aligned}
$$


Then Bob's system suffers Eve's attack and the bipartite state becomes

$$
\begin{aligned}
\left|\varphi_{A B E}\right\rangle & =\left(I_{A} \otimes U_{B E}\right)\left[\left|\Psi^{(4+\theta) n}\right\rangle \otimes\left|e_{0}\right\rangle\right] \\
& =\frac{1}{\sqrt{2^{(4+\theta) n}}} \sum_{\boldsymbol{l} \in \mathbf{F}_{2}^{(4+\theta) n}} H^{[\boldsymbol{a}]}|\boldsymbol{l}\rangle \otimes U_{B E}\left(H^{[\boldsymbol{a}]}|\boldsymbol{l}\rangle \otimes\left|e_{0}\right\rangle\right),
\end{aligned}
$$

where $\operatorname{Tr}_{E}\left[\left|\varphi_{A B E}\right\rangle\left\langle\varphi_{A B E}\right|\right]=[\mathcal{I} \otimes \mathcal{A}]\left(\left|\Psi^{(4+\theta) n}\right\rangle\left\langle\Psi^{(4+\theta) n}\right|\right)$, and $\operatorname{Tr}_{E}[\cdot]$ denotes the partial trace over $\mathcal{H}_{E}$. After that, Alice measures her system by the $\{|0\rangle,|1\rangle\}$ basis or the $\{|+\rangle,|-\rangle\}$ basis according to $\boldsymbol{a}$, and obtain $\boldsymbol{k} \in \mathbf{F}_{2}^{(4+\theta) n}$. This measurement changes the state in Eq. (5) to

$$
H^{[\boldsymbol{a}]}|\boldsymbol{k}\rangle \otimes U_{B E}\left[H^{[\boldsymbol{a}]}|\boldsymbol{k}\rangle \otimes\left|e_{0}\right\rangle\right] .
$$

Then Bob measures his system in Eq. ([6]) by the $\{|0\rangle,|1\rangle\}$ basis or the $\{|+\rangle,|-\rangle\}$ basis according to $\boldsymbol{b}$, and obtain $\tilde{\boldsymbol{k}}$.

Let

$$
\mathcal{P}(\boldsymbol{c}, \boldsymbol{d})=\left\langle\Psi_{\text {cd }}^{(4+\theta) n}\left|[\mathcal{I} \otimes \mathcal{A}]\left(\left|\Psi^{(4+\theta) n}\right\rangle\left\langle\Psi^{(4+\theta) n}\right|\right)\right| \Psi_{\text {cd }}^{(4+\theta) n}\right\rangle,
$$

where $\boldsymbol{c}, \boldsymbol{d} \in \mathbf{F}_{2}^{(4+\theta) n}$. Since Alice and Bob measure the qubits by $\{|0\rangle,|1\rangle\}$ basis when $a_{i}=b_{i}=0$ and by $\{|+\rangle,|-\rangle\}$ basis when $a_{i}=b_{i}=1$, we can relate $\boldsymbol{c}, \boldsymbol{d}$ with $\boldsymbol{k}, \tilde{k}$ as

$$
\begin{gathered}
\boldsymbol{c}_{T_{0}^{t}}=\boldsymbol{k}_{T_{0}^{t}}-\tilde{\boldsymbol{k}}_{T_{0}^{t}}=\boldsymbol{f}_{0} \\
\boldsymbol{d}_{T_{1}^{t}}=\boldsymbol{k}_{T_{1}^{t}}-\tilde{\boldsymbol{k}}_{T_{1}^{t}}=\boldsymbol{f}_{1},
\end{gathered}
$$

where $\boldsymbol{f}_{0}$ and $\boldsymbol{f}_{1}$ are the $\frac{n}{2}$-bit strings from which we estimate error rates.

\section{A.5 Relating the BB84 protocol to the CSS code protocol}

In the BB84 protocol, we select the linear codes $C_{1}$ and $C_{2}$ that satisfy the

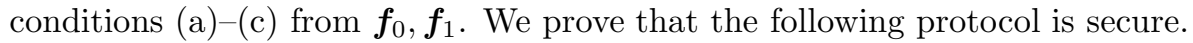

Alice randomly selects coset representatives $\boldsymbol{u}, \boldsymbol{x}, \boldsymbol{z}$, where $\boldsymbol{u} \in \pi\left(C_{1}\right) / \pi\left(C_{2}\right)$, $\boldsymbol{x} \in \mathbf{F}_{2}^{n} / \pi\left(C_{1}\right), \boldsymbol{z} \in \mathbf{F}_{2}^{n} / \pi\left(C_{2}^{\perp}\right)$, and $\boldsymbol{u}$ corresponds to a shared key in the BB84 protocol. Then Alice selects $|\boldsymbol{l}\rangle \otimes|\boldsymbol{m}\rangle$, where $\boldsymbol{l} \in \mathbf{F}_{2}^{n}, \boldsymbol{m} \in \mathbf{F}_{2}^{(2+\theta) n}, \boldsymbol{l}$ corresponds to test bits, and $\boldsymbol{m}$ corresponds to discarded bits in the BB84 protocol. Then Alice sends

$$
H^{[a]}\left[\left|\phi_{u x z}\right\rangle \otimes|\boldsymbol{l}\rangle \otimes|\boldsymbol{m}\rangle\right]
$$

to Bob, where $H^{[\boldsymbol{a}]}=H^{a_{1}} \otimes \cdots \otimes H^{a_{(4+\theta) n}}$. Bob measures the test qubits, and Alice and Bob obtain $\boldsymbol{f}_{0}, \boldsymbol{f}_{1}$. Then, Bob corrects errors and obtain a key. state

We consider this procedure as follows. First, Alice and Bob share a bipartite

$$
\rho=\left|\Phi_{\boldsymbol{x} \boldsymbol{z}}^{\prime}\right\rangle\left\langle\Phi_{\boldsymbol{x} \boldsymbol{z}}^{\prime}|\otimes| \Psi^{n}\right\rangle\left\langle\Psi^{n}|\otimes| \Psi^{(2+\theta) n}\right\rangle\left\langle\Psi^{(2+\theta) n}\right|,
$$


where

$$
\left|\Phi_{\boldsymbol{x} \boldsymbol{z}}^{\prime}\right\rangle=\left[H^{\left[\boldsymbol{a}_{T^{k}}\right]} \otimes H^{\left[\boldsymbol{a}_{T^{k}}\right]}\right]\left|\Phi_{\boldsymbol{x} \boldsymbol{z}}\right\rangle
$$

Then, Bob's system suffers a Eve's attack $\mathcal{A}$, and $\rho$ becomes $\rho^{\prime}$. After Alice and Bob obtain measurement disagreements $\boldsymbol{f}_{0}, \boldsymbol{f}_{1}$, bipartite state is

$$
\rho^{\prime \prime}=\frac{\left[I^{\otimes 2 n} \otimes \Pi\left(\boldsymbol{f}_{0}, \boldsymbol{f}_{1}\right) \otimes I^{\otimes(4+2 \theta) n}\right] \rho^{\prime}\left[I^{\otimes 2 n} \otimes \Pi\left(\boldsymbol{f}_{0}, \boldsymbol{f}_{1}\right) \otimes I^{\otimes(4+2 \theta) n}\right]}{T r\left[\left[I^{\otimes 2 n} \otimes \Pi\left(\boldsymbol{f}_{0}, \boldsymbol{f}_{1}\right) \otimes I^{\otimes(4+2 \theta) n}\right] \rho^{\prime}\right]},
$$

where

$$
\begin{aligned}
\Pi\left(\boldsymbol{f}_{0}, \boldsymbol{f}_{1}\right) & =\sum_{\boldsymbol{g}_{0}, \boldsymbol{g}_{1} \in \mathbf{F}_{2}^{\frac{n}{2}}}\left|\Psi_{\boldsymbol{f}_{0} \boldsymbol{f}_{1}}\right\rangle\left\langle\Psi_{\boldsymbol{f}_{0} \boldsymbol{f}_{1} \mid}\right. \\
\left|\Psi_{\boldsymbol{f}_{0} \boldsymbol{f}_{1}}\right\rangle & =\frac{1}{\sqrt{2^{n}}} \sum_{\boldsymbol{l} \in \mathbf{F}_{2}^{n}}|\boldsymbol{l}\rangle \otimes\left[\sigma_{x}^{\left[\boldsymbol{f}_{0}\right]} \sigma_{z}^{\left[\boldsymbol{g}_{0}\right]} \otimes \sigma_{x}^{\left[\boldsymbol{g}_{1}\right]} \sigma_{z}^{\left[\boldsymbol{f}_{1}\right]}\right]|\boldsymbol{l}\rangle
\end{aligned}
$$

Then Bob perform the recovery operation $\mathcal{R}_{\boldsymbol{x} \boldsymbol{z}}$, and we have

$$
\rho^{\prime \prime \prime}=\left[\mathcal{I}_{n} \otimes \mathcal{R}_{\boldsymbol{x} \boldsymbol{z}} \otimes \mathcal{I}_{(6+2 \theta) n}\right]\left(\rho^{\prime \prime}\right)
$$

We define the entanglement fidelity of the system that corresponds to a shared key as

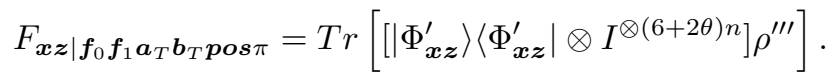

To bound the mutual information between a shared key and Eve's eavesdropping key, we evaluate Eq. (7) as follows. Since we transmit the qubits in the $\{|+\rangle,|-\rangle\}$ basis when $a_{i}=1$, and from Eq. (4), we can bound Eq. (7) as

$$
\begin{aligned}
& 1-\mathbb{E}_{\boldsymbol{x} \boldsymbol{z}} F_{\boldsymbol{x} \boldsymbol{z} \mid \boldsymbol{f}_{0} \boldsymbol{f}_{1} \boldsymbol{a}_{T} \boldsymbol{b}_{T} \boldsymbol{p o s} \pi} \leq \sum_{\left(\boldsymbol{c}_{T^{k}}, \boldsymbol{d}_{T^{k}}\right) \in \mathcal{E}} \mathcal{P}\left(\boldsymbol{c}_{T^{k}}, \boldsymbol{d}_{T^{k}} \mid \boldsymbol{f}_{0}, \boldsymbol{f}_{1}\right) \\
& \leq \sum_{\left(\boldsymbol{c}_{T_{0}^{k}}, \boldsymbol{d}_{T_{1}^{k}}\right) \in \mathcal{E}\left(\pi\left(C_{1}\right)\right)} \mathcal{P}\left(\boldsymbol{c}_{T_{0}^{k}}, \boldsymbol{d}_{T_{1}^{k}} \mid \boldsymbol{f}_{0}, \boldsymbol{f}_{1}\right) \\
&+\sum_{\left(\boldsymbol{c}_{T_{1}^{k}}, \boldsymbol{d}_{T_{0}^{k}}\right) \in \mathcal{E}\left(\pi\left(C_{2}^{\perp}\right)\right)} \mathcal{P}\left(\boldsymbol{c}_{T_{1}^{k}}, \boldsymbol{d}_{T_{0}^{k}} \mid \boldsymbol{f}_{0}, \boldsymbol{f}_{1}\right),
\end{aligned}
$$

where $\mathcal{E}$ is the set of uncorrectable errors by the CSS code that is constructed by $\pi\left(C_{1}\right)$ and $\pi\left(C_{2}\right)$, and $\mathcal{E}\left(\pi\left(C_{1}\right)\right), \mathcal{E}\left(\pi\left(C_{2}^{\perp}\right)\right)$ are sets of uncorrectable errors by $\pi\left(C_{1}\right)$ and $\pi\left(C_{2}^{\perp}\right)$ as a part of a CSS code respectively. Note that $\pi\left(C_{1}\right)$ corrects errors caused by $\sigma_{z}$ and $\pi\left(C_{2}^{\perp}\right)$ corrects errors caused by $\sigma_{x}$ in qubits transmitted by the $\{|+\rangle,|-\rangle\}$ basis, and that the second inequality is due to that the decoding error of a CSS code occurs when a bit flip error or a phase flip error is uncorrectable. 


\section{A.6 Bounding the fidelity}

In this section, we evaluate Eq. (8) by taking the average of parameters, $\boldsymbol{a}, \boldsymbol{b}, \pi, \boldsymbol{f}_{0}, \boldsymbol{f}_{1}$. Let fix one realization of $\left(\boldsymbol{c}_{T}, \boldsymbol{d}_{T}\right)$ and vary $\boldsymbol{a}_{T}, \boldsymbol{b}_{T}, \boldsymbol{p o s}$ at uniformly random. Note that subsequences $\boldsymbol{c}_{T_{0}^{k}}, \boldsymbol{c}_{T_{0}^{t}}, \boldsymbol{c}_{T_{1}^{k}}, \boldsymbol{c}_{T_{1}^{t}}, \boldsymbol{d}_{T_{0}^{k}}, \boldsymbol{d}_{T_{0}^{t}}, \boldsymbol{d}_{T_{1}^{k}}, \boldsymbol{d}_{T_{1}^{t}}$ of $\boldsymbol{c}_{T}, \boldsymbol{d}_{T}$ vary according to $\boldsymbol{a}_{T}, \boldsymbol{b}_{T}, \boldsymbol{p o s}$. Using a lemma [6, Lemma 5], we have

$$
\begin{gathered}
\operatorname{Pr}\left\{\left|Q_{\boldsymbol{c}_{T_{0}^{k}}}(1)-Q_{\boldsymbol{c}_{T_{0}^{t}}}(1)\right|>\delta \text { or }\left|Q_{\boldsymbol{d}_{T_{1}^{k}}}(1)-Q_{\boldsymbol{d}_{T_{1}^{t}}}(1)\right|>\delta\right\} \leq \exp \left\{-\Theta\left(\delta^{2} n\right)\right\} \\
\operatorname{Pr}\left\{\left|Q_{\boldsymbol{d}_{T_{0}^{k}}}(1)-Q_{\boldsymbol{d}_{T_{1}^{t}}}(1)\right|>\delta \text { or }\left|Q_{\boldsymbol{c}_{T_{1}^{k}}}(1)-Q_{\boldsymbol{c}_{T_{0}^{t}}}(1)\right|>\delta\right\} \\
\leq \exp \left\{-\Theta\left(\delta^{2} n\right)\right\}
\end{gathered}
$$

where the base of $\exp (\cdot)$ is 2 , and $\Theta\left(\delta^{2} n\right)$ can be explicitly given as

$$
\Theta\left(\delta^{2} n\right)=\frac{\delta^{2}}{4 \ln 2} n-2 \log (n+1)-2 .
$$

For each realization $\boldsymbol{c}_{T_{0}^{t}}=\boldsymbol{f}_{0}, \boldsymbol{d}_{T_{1}^{t}}=\boldsymbol{f}_{1}$, we decide linear codes $C_{1}$ and $C_{2}$ that satisfy the conditions (远) $-($ ( $\mathbb{c})$.

First, we consider $C_{1}$. Assume that $C_{1}$ is used over a BSC whose crossover probability of first $\frac{n}{2}$ bits are $Q_{\boldsymbol{c}_{T_{0}}}(1)$ and that of latter $\frac{n}{2}$ bits are $Q_{\boldsymbol{d}_{T_{1}^{k}}}(1)$. From the condition (b), if $Q_{\boldsymbol{c}_{T_{0}}}(1) \leq Q_{\boldsymbol{f}_{0}}(1)+\delta$ and $Q_{\boldsymbol{d}_{T_{1}^{k}}}(1) \leq Q_{\boldsymbol{f}_{1}}(1)+\delta$, then the decoding error probability of $C_{1}$ as a part of a CSS code is lower than or equal to $\epsilon$. We can write the decoding error probability as

$$
\sum_{\left(P_{\zeta}, P_{\eta}\right) \in P_{\frac{n}{2}}^{2}} \epsilon_{\left(P_{\zeta}, P_{\eta}\right)} Q_{B S C}\left(T_{\left(P_{\zeta}, P_{\eta}\right)}^{n}\right)=\sum_{\boldsymbol{e} \in \mathcal{E}\left(C_{1}\right)} Q_{B S C}(\boldsymbol{e}) \leq \epsilon,
$$

where $Q_{B S C}(\boldsymbol{e})$ is a probability that $\boldsymbol{e}$ occurs over a BSC whose crossover probability of first $\frac{n}{2}$ bits are $Q_{c_{T_{0}^{k}}}(1)$ and that of latter $\frac{n}{2}$ bits are $Q_{\boldsymbol{d}_{T_{1}^{k}}}(1), \mathcal{E}\left(C_{1}\right)$ is a set of uncorrectable errors of $C_{1}$, and $\epsilon_{\left(P_{\zeta}, P_{\eta}\right)}$ is the ratio of uncorrectable errors in $T_{\left(P_{\zeta}, P_{\eta}\right)}^{n}$, i.e.,

$$
\epsilon_{\left(P_{\zeta}, P_{\eta}\right)}=\frac{\left|T_{\left(P_{\zeta}, P_{\eta}\right)}^{n} \cap \mathcal{E}\left(C_{1}\right)\right|}{\left|T_{\left(P_{\zeta}, P_{\eta}\right)}^{n}\right|}
$$

From Eq. (11), we have

$$
\epsilon_{\left(Q_{T_{0}^{k}}, Q_{d_{T_{1}^{k}}}\right)} Q_{B S C}\left(T_{\left(Q_{c_{T_{0}^{k}}}, Q_{\boldsymbol{d}_{T_{1}^{k}}}\right)}^{n}\right) \leq \epsilon
$$

Using type property in [9. Lemma 2.6], we have

$Q_{B S C}\left(T_{\left(Q_{T_{T_{0}^{k}}}\right.}^{n}, Q_{\boldsymbol{d}_{T_{1}^{k}}}\right) \geq \frac{1}{\left(\frac{n}{2}+1\right)^{2}} 2^{-n \frac{D\left(Q_{c_{T_{0}^{k}}} \mid Q_{T_{T_{0}^{k}}}\right)+D\left(Q_{\boldsymbol{d}_{T_{1}^{k}}} \mid Q_{\boldsymbol{d}_{T_{1}^{k}}}\right)}{2}}=\frac{1}{\left(\frac{n}{2}+1\right)^{2}}$. 
Thus, we have

$$
\epsilon_{\left(Q_{T_{0}^{k}}, Q_{d_{T_{1}^{k}}}\right)} \leq\left(\frac{n}{2}+1\right)^{2} \epsilon .
$$

Consequently, if $\left|Q_{\boldsymbol{c}_{T_{0}^{k}}}(1)-Q_{\boldsymbol{f}_{0}}(1)\right| \leq \delta$ and $\left|Q_{\boldsymbol{d}_{T_{1}^{k}}}(1)-Q_{\boldsymbol{f}_{1}}(1)\right| \leq \delta$, then the ratio of uncorrectable errors of $C_{1}$ in $T_{\left(Q_{c_{T_{0}^{k}}}, Q_{d_{T_{1}^{k}}}\right)}^{n}$ is less than or equal to $\left(\frac{n}{2}+1\right)^{2} \epsilon$.

Define $J\left(\boldsymbol{c}_{T_{0}^{k}}, \boldsymbol{d}_{T_{1}^{k}}, \boldsymbol{f}_{0}, \boldsymbol{f}_{1}, C_{1}\right)$ as follows: If $\left(\boldsymbol{c}_{T_{0}^{k}}, \boldsymbol{d}_{T_{1}^{k}}\right) \in \mathcal{E}\left(C_{1}\right), J\left(\boldsymbol{c}_{T_{0}^{k}}, \boldsymbol{d}_{T_{1}^{k}}, \boldsymbol{f}_{0}, \boldsymbol{f}_{1}, C_{1}\right)=$ 1. For the others, $J\left(\boldsymbol{c}_{T_{0}^{k}}, \boldsymbol{d}_{T_{1}^{k}}, \boldsymbol{f}_{0}, \boldsymbol{f}_{1}, C_{1}\right)=0$. If $Q_{\boldsymbol{f}_{0}}(1)$ or $Q_{\boldsymbol{f}_{1}}(1)$ is too large and we abort the BB84 protocol, then $J\left(\boldsymbol{c}_{T_{0}^{k}}, \boldsymbol{d}_{T_{1}^{k}}, \boldsymbol{f}_{0}, \boldsymbol{f}_{1}, C_{1}\right)$ is always 0 . Note that $C_{1}$ is decided from $\boldsymbol{f}_{0}, \boldsymbol{f}_{1}$. From Eq. (9), $\left|Q_{T_{0}^{k}}(1)-Q_{\boldsymbol{f}_{0}}(1)\right| \leq \delta$ and $\left|Q_{T_{1}^{k}}(1)-Q_{\boldsymbol{f}_{1}}(1)\right| \leq \delta$ with high probability. When $\boldsymbol{e}, \boldsymbol{e}^{\prime} \in T_{\left(P_{\zeta}, P_{\eta}\right)}^{n}$, there exist a permutation $\pi$ such that $\pi(\boldsymbol{e})=\boldsymbol{e}^{\prime}$. Thus, if we consider the decoding error probability of $\pi\left(C_{1}\right)$ averaged over permutation $\pi$, then we can consider that an error with the same type occurs with same the probability. We proved that if $\left|Q_{T_{0}^{k}}(1)-Q_{f_{0}}(1)\right| \leq \delta$ and $\left|Q_{T_{1}^{k}}(1)-Q_{\boldsymbol{f}_{1}}(1)\right| \leq \delta$, then the ratio of uncorrectable errors of $\pi\left(C_{1}\right)$ in $T_{\left(Q_{T_{T_{0}^{k}}}, Q_{d_{T_{1}^{k}}}\right)}$ is less than or equal to $\left(\frac{n}{2}+1\right)^{2} \epsilon$ in Eq. (12).

Then we have

$$
\begin{aligned}
& \mathbb{E}_{\boldsymbol{a}_{T}, \boldsymbol{b}_{T}, \boldsymbol{p o s}, \pi} J\left(\boldsymbol{c}_{T_{0}^{k}}, \boldsymbol{d}_{T_{1}^{k}}, \boldsymbol{f}_{0}, \boldsymbol{f}_{1}, \pi\left(C_{1}\right)\right) \\
\leq & \left(\frac{n}{2}+1\right)^{2} \epsilon \operatorname{Pr}\left\{\left|Q_{\boldsymbol{c}_{T_{0}^{k}}}(1)-Q_{\boldsymbol{c}_{T_{0}^{t}}}(1)\right| \leq \delta \text { and }\left|Q_{\boldsymbol{d}_{T_{1}^{k}}}(1)-Q_{\boldsymbol{d}_{T_{1}^{t}}}(1)\right| \leq \delta\right\} \\
+ & \operatorname{Pr}\left\{\left|Q_{\boldsymbol{c}_{T_{0}^{k}}}(1)-Q_{\boldsymbol{c}_{T_{0}^{t}}}(1)\right|>\delta \text { or }\left|Q_{\boldsymbol{d}_{T_{1}^{k}}}(1)-Q_{\boldsymbol{d}_{T_{1}^{t}}}(1)\right|>\delta\right\} \\
\leq & \left(\frac{n}{2}+1\right)^{2} \epsilon+\exp \left\{-\Theta\left(\delta^{2} n\right)\right\} .
\end{aligned}
$$

Taking the average of Eq. (13) over $\left(\boldsymbol{c}_{T}, \boldsymbol{d}_{T}\right)$ and exchanging the order of the averages, we have

$$
\begin{aligned}
& \mathbb{E}_{\boldsymbol{a}_{T}, \boldsymbol{b}_{T}, \boldsymbol{p o s}, \pi} \mathbb{E}_{\mathcal{P}\left(\boldsymbol{f}_{0}, \boldsymbol{f}_{1}\right)} \sum_{\left(\boldsymbol{c}_{T_{0}^{k}}, \boldsymbol{d}_{T_{1}^{k}}\right) \in \mathcal{E}\left(\pi\left(C_{1}\right)\right)} \mathcal{P}\left(\boldsymbol{c}_{T_{0}^{k}}, \boldsymbol{d}_{T_{1}^{k}} \mid \boldsymbol{f}_{0}, \boldsymbol{f}_{1}\right) \\
= & \mathbb{E}_{\mathcal{P}\left(\boldsymbol{c}_{T}, \boldsymbol{d}_{T}\right)} \mathbb{E}_{\boldsymbol{a}_{T}, \boldsymbol{b}_{T}, \boldsymbol{p o s}, \pi} J\left(\boldsymbol{c}_{T_{0}^{k}}, \boldsymbol{d}_{T_{1}^{k}}, \boldsymbol{f}_{0}, \boldsymbol{f}_{1}, \pi\left(C_{1}\right)\right) \\
\leq & \left(\frac{n}{2}+1\right)^{2} \epsilon+\exp \left\{-\Theta\left(\delta^{2} n\right)\right\} .
\end{aligned}
$$

In the same way, we have

$$
\mathbb{E}_{\boldsymbol{a}_{T}, \boldsymbol{b}_{T}, \boldsymbol{p o s}, \pi} \mathbb{E}_{\mathcal{P}\left(\boldsymbol{f}_{0}, \boldsymbol{f}_{1}\right)} \sum_{\left(\boldsymbol{c}_{T_{1}^{k}}, \boldsymbol{d}_{T_{0}^{k}}\right) \in \mathcal{E}\left(\pi\left(C_{2}^{\perp}\right)\right)} \mathcal{P}\left(\boldsymbol{c}_{T_{1}^{k}}, \boldsymbol{d}_{T_{0}^{k}} \mid \boldsymbol{f}_{0}, \boldsymbol{f}_{1}\right) \leq\left(\frac{n}{2}+1\right)^{2} \epsilon+\exp \left\{-\Theta\left(\delta^{2} n\right)\right\} .
$$

From Eq. (14) and (15), we can rewrite Eq. (8) as

$$
1-\mathbb{E}_{\boldsymbol{a}_{T}, \boldsymbol{b}_{T}, \boldsymbol{p o s}, \pi} \mathbb{E}_{\mathcal{P}\left(\boldsymbol{f}_{0}, \boldsymbol{f}_{1}\right)} \mathbb{E}_{\boldsymbol{x} \boldsymbol{z}} F_{\boldsymbol{x} \boldsymbol{z} \mid \boldsymbol{f}_{0} \boldsymbol{f}_{1} \boldsymbol{a}_{T} \boldsymbol{b}_{T} \boldsymbol{p o s} \pi} \leq 2\left(\frac{n}{2}+1\right)^{2} \epsilon+2 \exp \left\{-\Theta\left(\delta^{2} n\right)\right\} .
$$




\section{A.7 Bounding the mutual information}

Using Eq. (3), we can bound the mutual information as

$$
\begin{aligned}
& \mathrm{I}\left(\boldsymbol{U} ; \boldsymbol{E} \mid \boldsymbol{X}, \boldsymbol{Z}, \boldsymbol{A}_{T}, \boldsymbol{B}_{T}, \boldsymbol{P O S}, \boldsymbol{\Pi}, \boldsymbol{F}_{0}, \boldsymbol{F}_{1}\right) \\
\leq & \mathbb{E}_{\boldsymbol{a}_{T}, \boldsymbol{b}_{T}, \boldsymbol{p o s}, \pi} \mathbb{E}_{\mathcal{P}\left(\boldsymbol{f}_{0}, \boldsymbol{f}_{1}\right)} \mathrm{H}\left(1-\mathbb{E}_{\boldsymbol{x} \boldsymbol{z}} F_{\left.\boldsymbol{x} \boldsymbol{z} \mid \boldsymbol{f}_{0} \boldsymbol{f}_{1} \boldsymbol{a}_{T} \boldsymbol{b}_{T} \boldsymbol{p o s} \pi\right)}\right) \\
& +\mathbb{E}_{\boldsymbol{a}_{T}, \boldsymbol{b}_{T}, \boldsymbol{p o s}, \pi} \mathbb{E}_{\mathcal{P}\left(\boldsymbol{f}_{0}, \boldsymbol{f}_{1}\right)}\left(1-\mathbb{E}_{\boldsymbol{x} \boldsymbol{z}} F_{\boldsymbol{x} \boldsymbol{z} \mid \boldsymbol{f}_{0} \boldsymbol{f}_{1} \boldsymbol{a}_{T} \boldsymbol{b}_{T} \boldsymbol{p o s} \pi}\right) 2 n \\
\leq & \mathrm{H}\left(1-\mathbb{E}_{\boldsymbol{a}_{T}, \boldsymbol{b}_{T}, \boldsymbol{p o s}, \pi} \mathbb{E}_{\mathcal{P}\left(\boldsymbol{f}_{0}, \boldsymbol{f}_{1}\right)} \mathbb{E}_{\boldsymbol{x} \boldsymbol{z} \boldsymbol{z}} F_{\left.\boldsymbol{x} \boldsymbol{z} \mid \boldsymbol{f}_{0} \boldsymbol{f}_{1} \boldsymbol{a}_{T} \boldsymbol{b}_{T} \boldsymbol{p o s} \pi\right)}\right) \\
& +\left(1-\mathbb{E}_{\boldsymbol{a}_{T}, \boldsymbol{b}_{T}, \boldsymbol{p o s}, \pi} \mathbb{E}_{\mathcal{P}\left(\boldsymbol{f}_{0}, \boldsymbol{f}_{1}\right)} \mathbb{E}_{\boldsymbol{x} \boldsymbol{z} \boldsymbol{z} \boldsymbol{x} \mid \boldsymbol{f}_{0} \boldsymbol{f}_{1} \boldsymbol{a}_{T} \boldsymbol{b}_{T} \boldsymbol{p o s} \pi}\right) 2 n \\
\leq & \mathrm{H}\left(2\left(\frac{n}{2}+1\right)^{2} \epsilon+2 \exp \left\{-\Theta\left(\delta^{2} n\right)\right\}\right) \\
& +4 n\left(\frac{n}{2}+1\right)^{2} \epsilon+4 n \exp \left\{-\Theta\left(\delta^{2} n\right)\right\},
\end{aligned}
$$

where $\boldsymbol{X}, \boldsymbol{Z}, \boldsymbol{A}_{T}, \boldsymbol{B}_{T}, \boldsymbol{P O S}, \boldsymbol{\Pi}, \boldsymbol{F}_{0}, \boldsymbol{F}_{1}$ denote the random variables of $\boldsymbol{x}, \boldsymbol{z}$, $\boldsymbol{a}_{T}, \boldsymbol{b}_{T}, \boldsymbol{p o s}, \pi, \boldsymbol{f}_{0}, \boldsymbol{f}_{1}$. Using the chain rule of mutual information 13. Theorem 2.5.2] and mutual independence of $\boldsymbol{U}$ from $\boldsymbol{X}, \boldsymbol{Z}, \boldsymbol{A}_{T}, \boldsymbol{B}_{T}, \boldsymbol{P O S}, \boldsymbol{\Pi}, \boldsymbol{F}_{0}, \boldsymbol{F}_{1}$, we can upper bound the mutual information of the shared key and Eve's all accessible information as

$$
\begin{aligned}
& \mathrm{I}\left(\boldsymbol{U} ; \boldsymbol{E}, \boldsymbol{X}, \boldsymbol{A}_{T}, \boldsymbol{B}_{T}, \boldsymbol{P O S}, \boldsymbol{\Pi}, \boldsymbol{F}_{0}, \boldsymbol{F}_{1}\right) \\
\leq & \mathrm{I}\left(\boldsymbol{U} ; \boldsymbol{E}, \boldsymbol{X}, \boldsymbol{Z}, \boldsymbol{A}_{T}, \boldsymbol{B}_{T}, \boldsymbol{P O S}, \boldsymbol{\Pi}, \boldsymbol{F}_{0}, \boldsymbol{F}_{1}\right) \\
= & \mathrm{I}\left(\boldsymbol{U} ; \boldsymbol{E} \mid \boldsymbol{X}, \boldsymbol{Z}, \boldsymbol{A}_{T}, \boldsymbol{B}_{T}, \boldsymbol{P O S}, \boldsymbol{\Pi}, \boldsymbol{F}_{0}, \boldsymbol{F}_{1}\right) \\
\leq & \mathrm{H}\left(2\left(\frac{n}{2}+1\right)^{2} \epsilon+2 \exp \left\{-\Theta\left(\delta^{2} n\right)\right\}\right)+4 n\left(\frac{n}{2}+1\right)^{2} \epsilon+4 n \exp \left\{-\Theta\left(\delta^{2} n\right)\right\},
\end{aligned}
$$

where $\Theta\left(\delta^{2} n\right)$ is given by Eq. (10).

\section{B Proof of lemma 4}

In this appendix, we prove

$$
\min _{\substack{0 \leq p_{0}^{\prime} \leq p_{0} \\ 0 \leq p_{1}^{\prime} \leq p_{1}}} E\left(R, p_{0}^{\prime}, p_{1}^{\prime}\right)=E\left(R, p_{0}, p_{1}\right),
$$

where we assume $p_{0}<\frac{1}{2}$ and $p_{1}<\frac{1}{2}$. First, we fix $p_{0}^{\prime}$ and $p_{1}^{\prime}$ arbitrary in the range $0 \leq p_{0}^{\prime} \leq p_{0}, 0 \leq p_{1}^{\prime} \leq p_{1}$, and analyze $E\left(R, p_{0}^{\prime}, p_{1}^{\prime}\right)$ as a function of $R$ in Section B.1] Then we prove that $E\left(R, p_{0}^{\prime}, p_{1}^{\prime}\right)$ takes the minimum at $p_{0}^{\prime}=p_{0}$, $p_{1}^{\prime}=p_{1}$ for arbitrary $0 \leq R \leq 1$ in Section B.2

\section{B.1 Analysis of $E\left(R, p_{0}^{\prime}, p_{1}^{\prime}\right)$}

Note that $p_{0}^{\prime}$ and $p_{1}^{\prime}$ are arbitrary fixed in the range $0 \leq p_{0}^{\prime} \leq p_{0}, 0 \leq p_{1}^{\prime} \leq p_{1}$ in this section. To express $E\left(R, p_{0}^{\prime}, p_{1}^{\prime}\right)$ as a function of $R, p_{0}^{\prime}, p_{1}^{\prime}$ explicitly, we define

$F\left(R, q_{0}, q_{1}\right)= \begin{cases}\frac{D\left(q_{0} \mid p_{0}^{\prime}\right)+D\left(q_{1} \mid p_{1}^{\prime}\right)}{2}+1-R-\frac{\mathrm{H}\left(q_{0}\right)+\mathrm{H}\left(q_{1}\right)}{2} & \text { for } \frac{\mathrm{H}\left(q_{0}\right)+\mathrm{H}\left(q_{1}\right)}{2}<1-R \\ \frac{D\left(q_{0} \mid p_{0}^{\prime}\right)+D\left(q_{1} \mid p_{1}^{\prime}\right)}{2} & \text { for } \frac{\mathrm{H}\left(q_{0}\right)+\mathrm{H}\left(q_{1}\right)}{2} \geq 1-R .\end{cases}$ 
Because it is obvious that $E\left(R, p_{0}^{\prime}, p_{1}^{\prime}\right)=0$ for $R \geq 1-\frac{\mathrm{H}\left(p_{0}^{\prime}\right)+\mathrm{H}\left(p_{1}^{\prime}\right)}{2}$, we assume $R<1-\frac{\mathrm{H}\left(p_{0}^{\prime}\right)+\mathrm{H}\left(p_{1}^{\prime}\right)}{2}$ in Section B.1.

First, we consider the case $\frac{\mathrm{H}\left(q_{0}\right)+\mathrm{H}\left(q_{1}\right)}{2}<1-R$. If we set

$$
\begin{aligned}
& q_{0}^{*}=\frac{\sqrt{p_{0}^{\prime}}}{\sqrt{p_{0}^{\prime}}+\sqrt{1-p_{0}^{\prime}}}, \\
& q_{1}^{*}=\frac{\sqrt{p_{1}^{\prime}}}{\sqrt{p_{1}^{\prime}}+\sqrt{1-p_{1}^{\prime}}},
\end{aligned}
$$

then we have $\left.\frac{\partial F\left(R, q_{0}, q_{1}^{*}\right)}{\partial q_{0}}\right|_{q_{0}=q_{0}^{*}}=0,\left.\frac{\partial F\left(R, q_{0}^{*}, q_{1}\right)}{\partial q_{1}}\right|_{q_{1}=q_{1}^{*}}=0$, and $\frac{\partial^{2} F\left(R, q_{0}, q_{1}\right)}{\partial q_{0}^{2}}>$ $0, \frac{\partial^{2} F\left(R, q_{0}, q_{1}\right)}{\partial q_{1}^{2}}>0$ for $0 \leq q_{0}, q_{1} \leq 1$. Thus, if $\frac{\mathrm{H}\left(q_{0}^{*}\right)+\mathrm{H}\left(q_{1}^{*}\right)}{2}<1-R$, then $F\left(R, q_{0}, q_{1}\right)$ takes the minimum at $\left(q_{0}^{*}, q_{1}^{*}\right)$ and we have

$$
E\left(R, p_{0}^{\prime}, p_{1}^{\prime}\right)=1-R-\log \left\{\sqrt{p_{0}^{\prime}}+\sqrt{1-p_{0}^{\prime}}\right\}-\log \left\{\sqrt{p_{1}^{\prime}}+\sqrt{1-p_{1}^{\prime}}\right\} .
$$

Next, we consider the case $\frac{\mathrm{H}\left(q_{0}^{*}\right)+\mathrm{H}\left(q_{1}^{*}\right)}{2} \geq 1-R . F\left(R, q_{0}, q_{1}\right)$ takes the minimum in the range $\frac{\mathrm{H}\left(q_{0}\right)+\mathrm{H}\left(q_{1}\right)}{2} \geq 1-R$. Because we assumed $R<1-\frac{\mathrm{H}\left(p_{0}^{\prime}\right)+\mathrm{H}\left(p_{1}^{\prime}\right)}{2}$, $q_{0}$ and $q_{1}$ must be $q_{0}>p_{0}^{\prime}$ or $q_{1}>p_{1}^{\prime}$ in order to satisfy $\frac{\mathrm{H}\left(q_{0}\right)+\mathrm{H}\left(q_{1}\right)}{2} \geq 1-R$. If $q_{0}<p_{0}^{\prime}$ and $q_{1}>p_{1}^{\prime}$, then $\frac{D\left(q_{0} \mid p_{0}^{\prime}\right)+D\left(q_{1} \mid p_{1}^{\prime}\right)}{2}$ can be smaller by taking larger $q_{0}$ and smaller $q_{1}$ while maintaining the condition $\frac{\mathrm{H}\left(q_{0}\right)+\mathrm{H}\left(q_{1}\right)}{2} \geq 1-R$. If $q_{0}>p_{0}^{\prime}$ and $q_{1}<p_{1}^{\prime}$, then $\frac{D\left(q_{0} \mid p_{0}^{\prime}\right)+D\left(q_{1} \mid p_{1}^{\prime}\right)}{2}$ can be smaller by taking larger $q_{1}$ and smaller $q_{0}$ while maintaining the condition $\frac{\mathrm{H}\left(q_{0}\right)+\mathrm{H}\left(q_{1}\right)}{2} \geq 1-R$. Thus, $q_{0}$ and $q_{1}$ must be $q_{0} \geq p_{0}^{\prime}$ and $q_{1} \geq p_{1}^{\prime}$ for $\frac{D\left(q_{0} \mid p_{0}^{\prime}\right)+D\left(q_{1} \mid p_{1}^{\prime}\right)}{2}$ to be the minimum. Thus $F\left(R, q_{0}, q_{1}\right)$ is minimum at $\left(q_{0}, q_{1}\right)$ that satisfy $\frac{\mathrm{H}\left(q_{0}\right)+\mathrm{H}\left(q_{1}\right)}{2}=1-R$. When $\frac{\mathrm{H}\left(q_{0}\right)+\mathrm{H}\left(q_{1}\right)}{2}=1-R$, we can expand $\frac{D\left(q_{0} \mid p_{0}^{\prime}\right)+D\left(q_{1} \mid p_{1}^{\prime}\right)}{2}$ as

$$
\begin{aligned}
& \frac{D\left(q_{0} \mid p_{0}^{\prime}\right)+D\left(q_{1} \mid p_{1}^{\prime}\right)}{2} \\
= & \frac{1}{2}\left\{-q_{0} \log p_{0}^{\prime}-\left(1-q_{0}\right) \log \left(1-p_{0}^{\prime}\right)\right. \\
& \left.-q_{1} \log p_{1}^{\prime}-\left(1-q_{1}\right) \log \left(1-p_{1}^{\prime}\right)-\mathrm{H}\left(q_{0}\right)-\mathrm{H}\left(q_{1}\right)\right\} \\
= & \frac{1}{2}\left\{\log \frac{1}{p_{0}^{\prime}}-\log \frac{1}{\left(1-p_{0}^{\prime}\right)}\right\} q_{0}+\frac{1}{2}\left\{\log \frac{1}{p_{1}^{\prime}}-\log \frac{1}{\left(1-p_{1}^{\prime}\right)}\right\} q_{1} \\
& +\frac{1}{2}\left\{\log \frac{1}{\left(1-p_{0}^{\prime}\right)}+\log \frac{1}{\left(1-p_{1}^{\prime}\right)}\right\}-(1-R) .
\end{aligned}
$$

Because

$$
\frac{d \mathrm{H}\left(q_{0}\right)}{d q_{0}}=\log \frac{1}{q_{0}}-\log \frac{1}{\left(1-q_{0}\right)},
$$

the tangent of the curve

$$
\frac{\mathrm{H}\left(q_{0}\right)+\mathrm{H}\left(q_{1}\right)}{2}=1-R
$$


at $\left(\hat{q}_{0}, \hat{q}_{1}\right)$ is

$$
\frac{1}{2}\left\{\log \frac{1}{\hat{q}_{0}}-\log \frac{1}{\left(1-\hat{q}_{0}\right)}\right\}\left(q_{0}-\hat{q}_{0}\right)+\frac{1}{2}\left\{\log \frac{1}{\hat{q}_{1}}-\log \frac{1}{\left(1-\hat{q}_{1}\right)}\right\}\left(q_{1}-\hat{q}_{1}\right)=0 .
$$

As is shown in Eq. (18), the curve

$$
\frac{D\left(q_{0} \mid p_{0}^{\prime}\right)+D\left(q_{1} \mid p_{1}^{\prime}\right)}{2}=\alpha
$$

is linear under the condition $\frac{\mathrm{H}\left(q_{0}\right)+\mathrm{H}\left(q_{1}\right)}{2}=1-R$, and $\alpha$ takes the minimum at the point where curve (19) and line (20) touch each other under the condition $\frac{\mathrm{H}\left(q_{0}\right)+\mathrm{H}\left(q_{1}\right)}{2}=1-R$. Then, $\left(\hat{q}_{0}, \hat{q}_{1}\right)$ satisfy

$$
\frac{\left\{\log \frac{1}{p_{1}^{\prime}}-\log \frac{1}{\left(1-p_{1}^{\prime}\right)}\right\}}{\left\{\log \frac{1}{p_{0}^{\prime}}-\log \frac{1}{\left(1-p_{0}^{\prime}\right)}\right\}}=\frac{\left\{\log \frac{1}{\hat{q}_{1}}-\log \frac{1}{\left(1-\hat{q}_{1}\right)}\right\}}{\left\{\log \frac{1}{\hat{q}_{0}}-\log \frac{1}{\left(1-\hat{q}_{0}\right)}\right\}}
$$

and we can further rewrite

$$
\begin{aligned}
& \left\{\log \frac{1}{\hat{q}_{0}}-\log \frac{1}{\left(1-\hat{q}_{0}\right)}\right\}=\beta\left\{\log \frac{1}{p_{0}^{\prime}}-\log \frac{1}{\left(1-p_{0}^{\prime}\right)}\right\} \\
& \left\{\log \frac{1}{\hat{q}_{1}}-\log \frac{1}{\left(1-\hat{q}_{1}\right)}\right\}=\beta\left\{\log \frac{1}{p_{1}^{\prime}}-\log \frac{1}{\left(1-p_{1}^{\prime}\right)}\right\} .
\end{aligned}
$$

Thus, if we set

$$
\begin{aligned}
\hat{q}_{0} & =\frac{\left(p_{0}^{\prime}\right)^{\beta}}{\left(p_{0}^{\prime}\right)^{\beta}+\left(1-p_{0}^{\prime}\right)^{\beta}}, \\
\hat{q}_{1} & =\frac{\left(p_{1}^{\prime}\right)^{\beta}}{\left(p_{1}^{\prime}\right)^{\beta}+\left(1-p_{1}^{\prime}\right)^{\beta}}, \\
\frac{\mathrm{H}\left(\hat{q}_{0}\right)+\mathrm{H}\left(\hat{q}_{1}\right)}{2} & =1-R,
\end{aligned}
$$

then $F\left(R, q_{0}, q_{1}\right)$ takes the minimum at $\left(\hat{q}_{0}, \hat{q}_{1}\right)$. Because $\frac{d \hat{q}_{0}}{d \beta}<0$ and $\frac{d \hat{q}_{1}}{d \beta}<0$ for $p_{0}^{\prime}<\frac{1}{2}$ and $p_{1}^{\prime}<\frac{1}{2}, \hat{q}_{0}$ and $\hat{q}_{1}$ are decreasing functions of $\beta$. Because we assumed $1-R>\frac{\mathrm{H}\left(p_{0}^{\prime}\right)+\mathrm{H}\left(p_{1}^{\prime}\right)}{2}$, we have $\beta<1$. If $\beta<\frac{1}{2}$, then $\frac{\mathrm{H}\left(q_{0}^{*}\right)+\mathrm{H}\left(q_{1}^{*}\right)}{2}<$ $\frac{\mathrm{H}\left(\hat{q}_{0}\right)+\mathrm{H}\left(\hat{q}_{1}\right)}{2}=1-R$ and $F\left(R, \hat{q}_{0}, \hat{q}_{1}\right)$ takes the minimum at $\left(q_{0}^{*}, q_{1}^{*}\right)$. Thus, $\frac{1}{2} \leq \beta<1$.

Consequently, we can write $E\left(R, p_{0}^{\prime}, p_{1}^{\prime}\right)$ as

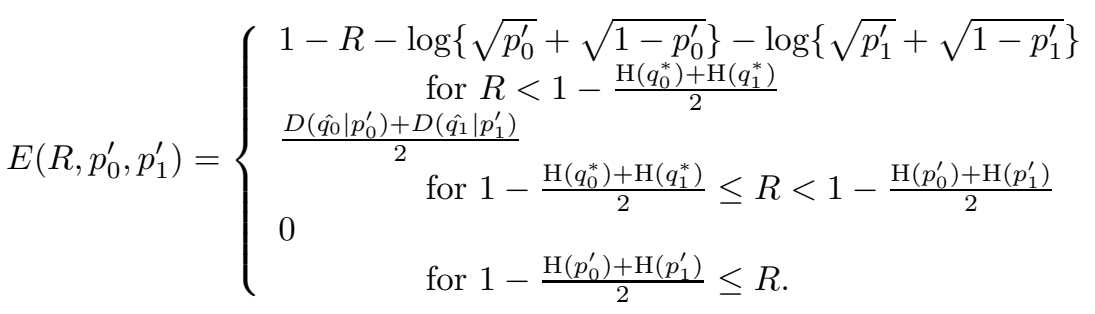




\section{B.2 The minimum of $E\left(R, p_{0}^{\prime}, p_{1}^{\prime}\right)$}

Next, we evaluate

$$
\min _{\substack{0 \leq p_{0}^{\prime} \leq p_{0} \\ 0 \leq p_{1}^{\prime} \leq p_{1}}} E\left(R, p_{0}^{\prime}, p_{1}^{\prime}\right)
$$

for arbitrary fixed rate $R$. If $\frac{\mathrm{H}\left(q_{0}^{*}\right)+\mathrm{H}\left(q_{1}^{*}\right)}{2}<1-R$,

$$
E\left(R, p_{0}^{\prime}, p_{1}^{\prime}\right)=1-R-\log \left\{\sqrt{p_{0}^{\prime}}+\sqrt{1-p_{0}^{\prime}}\right\}-\log \left\{\sqrt{p_{1}^{\prime}}+\sqrt{1-p_{1}^{\prime}}\right\} .
$$

Eq. (21) is a decreasing function of $p_{0}^{\prime}, p_{1}^{\prime}$ for $p_{0}^{\prime}<\frac{1}{2}, p_{1}^{\prime}<\frac{1}{2}$, because if we set $f\left(p_{0}^{\prime}\right)=\sqrt{p_{0}^{\prime}}+\sqrt{1-p_{0}^{\prime}}$,

$$
\frac{d f\left(p_{0}^{\prime}\right)}{d p_{0}^{\prime}}=\frac{1}{2}\left\{\frac{1}{\sqrt{p_{0}^{\prime}}}-\frac{1}{\sqrt{1-p_{0}^{\prime}}}\right\}>0
$$

for $p_{0}^{\prime}<\frac{1}{2}$ and $f\left(p_{0}^{\prime}\right)$ is an increasing function of $p_{0}^{\prime}$. If $\frac{\mathrm{H}\left(q_{0}^{*}\right)+\mathrm{H}\left(q_{1}^{*}\right)}{2} \geq 1-R$, then

$$
E\left(R, p_{0}^{\prime}, p_{1}^{\prime}\right)=\frac{D\left(\hat{q_{0}} \mid p_{0}^{\prime}\right)+D\left(\hat{q_{1}} \mid p_{1}^{\prime}\right)}{2} .
$$

Assume $E\left(R, p_{0}^{\prime}, p_{1}^{\prime}\right)$ takes the minimum at $\left(p_{0}^{\prime}, p_{1}^{\prime}\right)$ with $p_{0}^{\prime}<p_{0}$. Because $p_{0}^{\prime}<\hat{q_{0}}$, we define $\left(p_{0}^{\prime \prime}, p_{1}^{\prime \prime}\right)$ such that $p_{0}^{\prime}<p_{0}^{\prime \prime}<\hat{q_{0}}$ and $p_{1}^{\prime}=p_{1}^{\prime \prime}$. Then

$$
\begin{aligned}
E\left(R, p_{0}^{\prime}, p_{1}^{\prime}\right) & =\frac{D\left(\hat{q_{0}} \mid p_{0}^{\prime}\right)+D\left(\hat{q_{1}} \mid p_{1}^{\prime}\right)}{2} \\
& >\frac{D\left(\hat{q_{0}} \mid p_{0}^{\prime \prime}\right)+D\left(\hat{q_{1}} \mid p_{1}^{\prime \prime}\right)}{2} \\
& \geq \frac{D\left(\tilde{q}_{0} \mid p_{0}^{\prime \prime}\right)+D\left(\tilde{q}_{1} \mid p_{1}^{\prime \prime}\right)}{2}=E\left(R, p_{0}^{\prime \prime}, p_{1}^{\prime \prime}\right),
\end{aligned}
$$

where

$$
\begin{aligned}
\tilde{q}_{0} & =\frac{\left(p_{0}^{\prime \prime}\right)^{\beta}}{\left(p_{0}^{\prime \prime}\right)^{\beta}+\left(1-p_{0}^{\prime \prime}\right)^{\beta}} \\
\tilde{q}_{1} & =\frac{\left(p_{1}^{\prime \prime}\right)^{\beta}}{\left(p_{1}^{\prime \prime}\right)^{\beta}+\left(1-p_{1}^{\prime \prime}\right)^{\beta}} \\
\frac{\mathrm{H}\left(\tilde{q}_{0}\right)+\mathrm{H}\left(\tilde{q}_{1}\right)}{2} & =1-R .
\end{aligned}
$$

Note that the first inequality is due to that $p_{0}^{\prime \prime}$ is closer to $\hat{q_{0}}$ than $p_{0}^{\prime}$, and the second inequality is due to that $\left(\tilde{q}_{0}, \tilde{q}_{1}\right)$ is the point at which $\frac{D\left(q_{0} \mid p_{0}^{\prime \prime}\right)+D\left(q_{1} \mid p_{1}^{\prime \prime}\right)}{2}$ takes the minimum. Thus, $E\left(R, p_{0}^{\prime}, p_{1}^{\prime}\right)$ does not take the minimum at $\left(p_{0}^{\prime}, p_{1}^{\prime}\right)$ with $p_{0}^{\prime}<p_{0}$. In a similar manner, we can show that $E\left(R, p_{0}^{\prime}, p_{1}^{\prime}\right)$ does not take the minimum at $\left(p_{0}^{\prime}, p_{1}^{\prime}\right)$ with $p_{1}^{\prime}<p_{1}$. Consequently, we have

$$
\min _{\substack{0 \leq p_{0}^{\prime} \leq p_{0} \\ 0 \leq p_{1}^{\prime} \leq p_{1}}} E\left(R, p_{0}^{\prime}, p_{1}^{\prime}\right)=E\left(R, p_{0}, p_{1}\right) .
$$




\section{References}

[1] C. H. Bennett and G. Brassard, "Quantum cryptography: Public key distribution and coin tossing," Proceedings of IEEE International Conference on Computers, Systems and Signal Processing, Bangalore, India, pp. 175-179, 1984.

[2] P. W. Shor and J. Preskill, "Simple proof of security of the BB84 quantum key distribution protocol," Phys. Rev. Lett, vol. 85, no. 2, pp. 441-444, July 2000.

[3] R. Matsumoto and T. Uyematsu, "Lower bound for the quantum capacity of a discrete memoryless quantum channel," J. Math. Phys., vol. 43, no. 9, pp. 4391-4403, September 2002.

[4] M. Hamada, "Exponential lower bound on the highest fidelity achievable by quantum error-correcting codes," Phys. Rev. A, vol. 65, no. 5, pp. 0523051-4, April 2002.

[5] D. Mayers, "Unconditional security in quantum cryptography," Journal of the ACM, vol. 48, no. 3, pp. 351-406, May 2001.

[6] M. Hamada, "Reliability of Calderbank-Shor-Steane codes and security of quantum key distribution," J. Phys. A: Math. Gen., vol. 37, no. 34, pp. 8303-8328, Aug 2004.

[7] D. Gottesman and H.-K. Lo, "Proof of security of quantum key distribution with two-way classical communications," IEEE Trans. Inform. Theory, vol. 49, no. 2, pp 457-475, Feburuary 2003.

[8] X.-B. Wang, "Quantum key distribution with asymmetric channel noise", Los Alamos E-print archive quant-ph/0406099.

[9] I. Csiszár and J. Körner, "Information theory: Coding theorems for discrete memoryless systems", Akademiai Kiado, 1981.

[10] X.-B. Wang, "Quantum key distribution with two-qubit quantum codes," Phys. Rev. Lett, vol. 92, no. 7, pp. 077902, February 2004.

[11] H. F. Chau, "Practical scheme to share a secret key through a quantum channel with a $27.6 \%$ bit error rate," Phys. Rev. A, vol. 66, no. 6, pp. 060302, December 2002.

[12] B. Schumacher, "Sending entanglement through noisy quantum channels," Phys. Rev. A, vol. 54, no. 4, pp. 2614-2628, October 1996.

[13] T. M. Cover and J. A. Thomas, "Elements of information theory," NY: Wiley, 1991.

[14] H. -K. Lo, H. F. Chau and M. Ardehali, "Efficient quantum key distribution scheme and proof of its unconditional security", quant-ph/0011056, 2000. 
[15] D. Gottesman and J. Preskill, "Secure quantum key distribution using squeezed states", Phys. Rev. A, vol. 63, no. 2, pp022309, January 2001.

[16] H. -K. Lo, "Proof of unconditional security of six-state quantum key distribution scheme", Quant. Inform. Comput, vol. 1, no. 2, pp.81-94, 2001. 